\title{
Calculation of Norms of Bethe Wave Functions
}

\author{
V. E. Korepin* \\ Service de Physique Théorique, Division de la Physique, C.E.N.-Saclay. Orme des Merisiers, \\ F-91191 Gif-sur-Yvette Cedex, France
}

\begin{abstract}
A class of two dimensional completely integrable models of statistical mechanics and quantum field theory is considered. Eigenfunctions of the Hamiltonians are known for these models. Norms of these eigenfunctions in the finite box are calculated in the present paper. These models include in particular the quantum nonlinear Schrödinger equation and the Heisenberg $X X Z$ model.
\end{abstract}

\section{Introduction}

A lot of two dimensional models have been solved by means of the Bethe Ansatz, see for example [1-5]. The recently formulated quantum inverse scattering method (QISM) $[6,7]$ disclosed the algebraic nature of these solutions. An interesting problem is the study of perturbations of these models. Such a study requires the knowledge of the norms of eigenfunctions in the finite box. Gaudin studied this problem for the quantum nonlinear Schrödinger equation [8] and made the remarkable hypothesis that the norm of the eigenfunction is equal to some Jacobian. A similar formula for the norm of the eigenfunction in the Heisenberg $X X Z$ model was presented in [9] by Gaudin et al. Authors of [9] emphasized that the arguments given in their paper do not really constitute a proof. In the present paper these formulae are proved and a more general result is obtained. The norms are calculated for any exactly solvable models with the $R$ matrix either of the $X X X$ model or of the $X X Z$ Heisenberg models.

The contents of the paper are as follows. In Sect. 2 the main formulas of QISM are presented. The proof of the norm formula is long, so in Sects. 3 and 4 the final formulas are given. In Sect. 5 the explicit expression for the anticipated answer is examined. We prove that this expression is characterized uniquely by some of its properties. In the rest of the paper these properties are proved for the norms of Bethe wave functions themselves. As the calculation of the norm itself is

* Permanent address: LOMI, Fontanka 27, SU-191011 Leningrad, USSR 
complicated, the scalar product of two different wave functions is examined at first. So in Sect. 6 the description of the scalar product is presented. Note that our approach to the description of the scalar products is close to that of [10]. The properties of the scalar product announced in Sect. 6 are proved in Sect. 8. In the proof a special case of the 6 vertex model is used. This model is constructed in Sect. 7 and represents the main point of the paper. The knowledge we have of the properties of the scalar product permits us to demonstrate the necessary properties of the norm. So in Sects. 9 and 10 the proof of the formula for the norm, announced in Sect. 4, is completed.

\section{Notations}

First of all let us remind the reader of some notations of the QISM. Eigenfunctions of the Hamiltonian of the physical system are constructed by means of the monodromy matrix of an auxiliary linear problem $T(\lambda)$. In our case $T(\lambda)$ is a $2 \times 2$ matrix, its matrix elements being quantum operators, which depend on the spectral parameter $\lambda$ :

$$
T(\lambda)=\left(\begin{array}{ll}
A(\lambda) & B(\lambda) \\
C(\lambda) & D(\lambda)
\end{array}\right)
$$

Commutation relations between these matrix elements are given by the formula:

$$
R(\lambda, \mu)(T(\lambda) \otimes I)(I \otimes T(\mu))=(I \times T(\mu))(T(\lambda) \times I) R(\lambda, \mu) .
$$

Here $I$ is the unit $2 \times 2$ matrix, $R(\lambda, \mu)$ is a $4 \times 4$ matrix with $c$-number elements. Another way of writing (2.2) is

$$
R_{\alpha \beta}(\lambda, \mu) T_{\alpha}(\lambda) T_{\beta}(\mu)=T_{\beta}(\mu) T_{\alpha}(\lambda) R_{\alpha \beta}(\lambda, \mu) .
$$

This means that $T_{\alpha}(\lambda)$ is a $2 \times 2$ matrix which acts on the 2 dimensional space with index $\alpha$ and $T_{\beta}$ is a matrix which acts on the space with index $\beta(\alpha \neq \beta)$. The matrix $R_{\alpha \beta}$ acts in the tensor product of these two spaces. We shall deal with $R$ matrices of the following form:

$$
R(\lambda, \mu)=\left|\begin{array}{cccc}
f(\mu, \lambda) & 0 & 0 & 0 \\
0 & 1 & g(\mu, \lambda) & 0 \\
0 & g(\mu, \lambda) & 1 & 0 \\
0 & 0 & 0 & f(\mu, \lambda)
\end{array}\right|
$$

For models of the $X X Z$ type:

$$
f(\lambda, \mu)=\frac{\sinh (\lambda-\mu+2 i \eta)}{\sinh (\lambda-\eta)}, \quad g(\lambda, \mu)=\frac{i \sin 2 \eta}{\sinh (\lambda-\mu)} .
$$

For models of the $X X X$ type:

$$
f(\lambda, \mu)=(\lambda-\mu+i \kappa) /(\lambda-\mu), \quad g(\lambda, \mu)=i \kappa /(\lambda-\mu) .
$$

Here $\lambda, \mu$ are spectral parameters and $\eta, \kappa$ are coupling constants. It is known that (2.6) is the limiting case of (2.5). One must replace $\lambda, \mu, \eta$ in (2.5) by

$$
\varepsilon \lambda, \quad \varepsilon \mu, \quad \varepsilon \eta,
$$


and let $\varepsilon \rightarrow 0$. In the notation (2.3) the $R$ matrix (2.4), (2.5) looks like

$$
\begin{aligned}
R_{\alpha \beta}(\lambda, \mu)= & {[\sinh (\mu-\lambda+i \eta) / \sinh (\mu-\lambda)] \cos \eta \cdot I_{\alpha} I_{\beta} } \\
& +[i(\sin \eta) \cdot \cosh (\mu-\lambda+i \eta) / \sinh (\mu-\lambda)] \sigma_{\alpha}^{3} \sigma_{\beta}^{3} \\
& +i[\sin 2 \eta / \sinh (\mu-\lambda)]\left(\sigma_{\alpha}^{-} \sigma_{\beta}^{+}+\sigma_{\alpha}^{+} \sigma_{\beta}^{-}\right) ; \\
2 \sigma^{ \pm}= & \sigma^{1} \pm i \sigma^{2} .
\end{aligned}
$$

Here $\sigma_{\alpha, \beta}$ are the standard Pauli matrices in the spaces with indices $\alpha$ or $\beta$ respectively. Let us write down the commutation relations (2.3) for the $R$ matrix (2.8) explicitly:

$$
\begin{gathered}
{[A(\lambda)+D(\lambda), A(\mu)+D(\mu)]=0,} \\
{[B(\lambda), B(\mu)]=0, \quad[C(\lambda), C(\mu)]=0,} \\
A(\mu) B(\lambda)=f(\mu, \lambda) B(\lambda) A(\mu)+g(\lambda, \mu) B(\mu) A(\lambda), \\
D(\mu) B(\lambda)=f(\lambda, \mu) B(\lambda) D(\mu)+g(\mu, \lambda) B(\mu) D(\lambda), \\
C(\lambda) A(\mu)=f(\mu, \lambda) A(\mu) C(\lambda)+g(\lambda, \mu) A(\lambda) C(\mu), \\
C(\lambda) D(\mu)=f(\lambda, \mu) D(\mu) C(\lambda)+g(\mu, \lambda) D(\lambda) C(\mu), \\
{\left[C\left(\lambda^{C}\right), B\left(\lambda^{B}\right)\right]=g\left(\lambda^{C}, \lambda^{B}\right)\left\{A\left(\lambda^{C}\right) D\left(\lambda^{B}\right)-A\left(\lambda^{B}\right) D\left(\lambda^{C}\right)\right\}} \\
=g\left(\lambda^{C}, \lambda^{B}\right)\left\{D\left(\lambda^{B}\right) A\left(\lambda^{C}\right)-D\left(\lambda^{C}\right) A\left(\lambda^{B}\right)\right\} .
\end{gathered}
$$

Other important objects in QISM are the pseudovacuum $|0\rangle$ and the dual pseudovacuum $\langle 0|$. These are vectors in the quantum space with the following properties :

$$
\begin{aligned}
& C(\lambda)|0\rangle=0, \quad A(\lambda)|0\rangle=a(\lambda)|0\rangle, \quad D(\lambda)|0\rangle=d(\lambda)|0\rangle \\
& \langle 0| B(\lambda)=0, \quad\langle 0| A(\lambda)=a(\lambda)\langle 0|, \quad\langle 0| D(\lambda)=d(\lambda)\langle 0| .
\end{aligned}
$$

Here vacuum eigenvalues $a(\lambda)$ and $d(\lambda)$ are $c$ number functions. The space in which the operators $A(\lambda), B(\lambda), C(\lambda)$ and $D(\lambda)$ act is constructed in Appendix A. Note that $a(\lambda)$ and $d(\lambda)$ are arbitrary differentiable functions. The norms and scalar products in question are functionals of these $a(\lambda)$ and $d(\lambda)$. We shall vary $a(\lambda)$ and $d(\lambda)$ and study the dependence of scalar products on these functional arguments.

The Hamiltonian of the physical system in question is expressed by means of the transfer matrix $t(\mu)=A(\mu)+D(\mu)$. The Hamiltonian and the transfer matrix have common eigenfunctions which are constructed as follows. Put

$$
\psi_{N}\left(\left\{\lambda_{j}\right\}\right)=B\left(\lambda_{1}\right) \ldots B\left(\lambda_{N}\right)|0\rangle,
$$

and suppose that $\lambda_{j}$ satisfy the system of the transcendental equations (TE):

$$
\left[a\left(\lambda_{n}\right) / d\left(\lambda_{n}\right)\right] \prod_{\substack{j=1 \\ j \neq n}}^{N}\left[f\left(\lambda_{n}, \lambda_{j}\right) / f\left(\lambda_{j}, \lambda_{n}\right)\right]=1 .
$$

Then $\psi_{N}\left(\left\{\lambda_{j}\right\}\right)$ is an eigenfunction of $t(\mu)$ with the eigenvalue:

$$
\theta(\mu)=a(\mu) \prod_{j=1}^{N} f\left(\mu, \lambda_{j}\right)+d(\mu) \prod_{j=1}^{N} f\left(\lambda_{j} \mu\right) .
$$

Here $N$ is called the number of particles. 
Notice that

$$
\tilde{\psi}_{N}\left(\left\{\lambda_{j}\right\}\right)=\langle 0| C\left(\lambda_{1}\right) \ldots C\left(\lambda_{N}\right)
$$

is a dual eigenfunction for $t(\mu)$ :

$$
\tilde{\psi}_{N}\left(\left\{\lambda_{j}\right\}\right) \cdot t(\mu)=\theta(\mu) \tilde{\psi}_{N}\left(\left\{\lambda_{j}\right\}\right)
$$

with the same eigenvalue (2.19). Eigenfunctions (2.17), (2.20) can be called Bethe wave functions. We consider the case of all different $\lambda_{j}$. If two of them coincide $\left(\lambda_{1}=\lambda_{2}\right)$ the following phenomena take place. The limiting form $\left(\lambda_{1} \rightarrow \lambda_{2}\right)$ of the system (2.18) contains $(N-1)$ equations, the number of different $\lambda_{j}$ is also $N-1$, but an additional equation appears [11]:

$$
i \frac{d}{d \lambda_{1}} \ln \frac{a\left(\lambda_{1}\right)}{d\left(\lambda_{1}\right)}+i \sum_{j=3}^{N} \frac{d}{d \lambda_{1}} \ln \frac{f\left(\lambda_{1}, \lambda_{j}\right)}{f\left(\lambda_{j}, \lambda_{1}\right)}+4 \operatorname{ctg}(2 \eta)=0 .
$$

Since the number of equations exceeds the number of $\lambda_{j}$, the solution does not generally exist.

Finally let us present two remarks. First of all new variables

$$
\varphi_{K}=i \ln \left[a\left(\lambda_{K}\right) / d\left(\lambda_{K}\right)\right]+i \sum_{\substack{j=1 \\ j \neq K}}^{N} \ln \left[f\left(\lambda_{K}, \lambda_{j}\right) / f\left(\lambda_{j}, \lambda_{K}\right)\right] \quad K=1, \ldots, N
$$

are convenient. For example the system (2.18) takes the form

$$
\varphi_{K}=0(\bmod 2 \pi) \text {. }
$$

Secondly below we shall use the monodromy matrix

$$
T_{y}(\lambda)=\left(\begin{array}{cc}
y A(\lambda) & y B(\lambda) \\
y^{-1} C(\lambda) & y^{-1} D(\lambda)
\end{array}\right)
$$

which satisfies Eq. (2.2) for any complex $y$, and has the following vacuum eigenvalues

$$
a_{y}(\lambda)=y a(\lambda), \quad d_{y}(\lambda)=y^{-1} \cdot d(\lambda) .
$$

Now we are able to formulate the main result of the paper.

\section{Expression for the Norm in the $X X X$ Type Models}

For models with an $R$ matrix of the form given by (2.4), (2.6) the scalar product of an eigenfunction (2.17) and dual eigenfunction (2.20) is equal to

$$
\begin{aligned}
\left\langle 0\left|C\left(\lambda_{1}\right) \ldots C\left(\lambda_{N}\right) B\left(\lambda_{1}\right) \ldots B\left(\lambda_{N}\right)\right| 0\right\rangle= & \kappa^{N}\left\{\prod_{j=1}^{N} a\left(\lambda_{j}\right) d\left(\lambda_{j}\right)\right\} \\
& \cdot\left\{\prod_{j=1}^{N} \prod_{\substack{K=1 \\
j \neq K}}^{N} f\left(\lambda_{j}, \lambda_{K}\right)\right\}\langle 0 \mid 0\rangle \operatorname{det}_{N}\left\{\partial \varphi_{K} / \partial \lambda_{j}\right\} .
\end{aligned}
$$


Here the $\varphi_{K}$ are the variables (2.23) and the set of the $\lambda_{j}$ is a solution of the system (2.18) (2.6). The derivatives can be written in the explicit form

$$
\frac{\partial \varphi_{K}}{\partial \lambda_{j}}=\delta_{K j}\left\{i \frac{\partial}{\partial \lambda_{k}} \ln \frac{a\left(\lambda_{K}\right)}{d\left(\lambda_{K}\right)}+\sum_{l=1}^{N} \frac{2 \kappa}{\left(\lambda_{K}-\lambda_{l}\right)^{2}+\kappa^{2}}\right\}-\frac{2 \kappa}{\left(\lambda_{K}-\lambda_{j}\right)^{2}+\kappa^{2}} .
$$

Formula (3.1) can be used to calculate the norm of the eigenfunction (2.17) if the following properties are valid. Hermitian conjugation must act as follows:

$$
B^{+}(\bar{\lambda})= \pm C(\lambda), \quad|0\rangle^{+}=\langle 0|
$$

The set of $\lambda_{j}$ must be invariant under complex conjugation:

$$
\left\{\bar{\lambda}_{j}\right\}=\left\{\lambda_{j}\right\} \text {. }
$$

In this case by means of (2.10) we have

$$
\left\langle 0\left|B^{+}\left(\lambda_{1}\right) \ldots B^{+}\left(\lambda_{N}\right) B\left(\lambda_{1}\right) \ldots B\left(\lambda_{N}\right)\right| 0\right\rangle=( \pm 1)^{N}\left\langle 0\left|C\left(\lambda_{1}\right) \ldots C\left(\lambda_{N}\right) B\left(\lambda_{1}\right) \ldots B\left(\lambda_{N}\right)\right| 0\right\rangle .
$$

The formula (3.1) is similar to formula (4.1) given in the next section. We shall first prove the latter and then in the end of Sect. 10 we shall discuss how to pass to formula (3.1).

Several examples of the application of (3.1) may clarify matters. Consider for example the norms of the eigenfunctions for the quantum nonlinear Schrödinger equation. The model was treated by means of QISM in $[12,13]$. The Hamiltonian is equal to

$$
H=\int_{0}^{L} d x\left(\psi_{x}^{+} \psi_{x}+\kappa \psi^{+} \psi^{+} \psi \psi\right)\left[\psi(x), \psi^{+}(y)\right]=\delta(x-y) .
$$

The monodromy matrix has the following property:

$$
\sigma^{1} T^{+}(\bar{\lambda}) \sigma^{1}=T(\lambda), \quad B^{+}(\bar{\lambda})=C(\lambda)
$$

Here the cross means Hermitian conjugation of the quantum operators. It does not transpose $T(\lambda)$ as a $2 \times 2$ matrix. The commutation relation of $T(\lambda)$ is given by the $R$ matrix (2.4), (2.6). The pseudovacuum coincides with the usual Fock vacuum. The vacuum eigenvalues are equal to

$$
a(\lambda)=\exp \{-i \lambda L / 2\}, \quad d(\lambda)=\exp \{i \lambda L / 2\} .
$$

Properties (3.3) and (3.4) are valid. To write down the trace identities it is necessary to construct the following asymptotic expansion:

$$
\ln \left[(A(\lambda)+D(\lambda)) \exp _{\lambda \rightarrow i \infty}\{i \lambda L / 2\}\right]=\sum_{K=1}^{\infty} \lambda^{-K} C_{K}
$$

The Hamiltonian (3.6) is equal to

$$
H=(i \kappa)^{-1} C_{3}+C_{2}+i \kappa C_{1} / 6
$$


due to the results of [14]. The eigenvalues of $H$ are equal to $\sum \lambda_{j}^{2}$. The square of the norm of the eigenfunction (2.17) is equal to

$$
\begin{gathered}
\left\langle 0\left|B^{+}\left(\lambda_{1}\right) \ldots B^{+}\left(\lambda_{N}\right) B\left(\lambda_{1}\right) \ldots B\left(\lambda_{N}\right)\right| 0\right\rangle=\kappa^{N} \prod_{j>K=1}^{N}\left[1+\frac{\kappa^{2}}{\left(\lambda_{K}-\lambda_{j}\right)^{2}}\right] \\
\cdot \operatorname{det}_{N}\left\{\delta_{K j}\left[L+\sum_{l=1}^{N} \frac{2 \kappa}{\left(\lambda_{k}-\lambda_{l}\right)^{2}+\kappa^{2}}\right]-\frac{2 \kappa}{\left(\lambda_{K}-\lambda_{j}\right)^{2}+K^{2}}\right\}
\end{gathered}
$$

due to the formulas (3.1), (3.2) and (3.5). To obtain the norm of the coordinate wave function an explicitly known factor must be extracted, see [15]. When this is done one sees that the norm is equal to the determinant in (3.11). In this way we prove the hypothesis stated by Gaudin in [8].

The formula (3.1) can be applied to other models. For example norms in the $X X X$ model can be calculated. In $[16,17]$ the quantum lattice nonlinear Schrödinger equation was constructed. In this model the norms can also be calculated by means of (3.1).

\section{Expression for the Norm in the $X X Z$ Type Models}

For models with an $R$ matrix of the form given by (2.4), (2.5) or (2.8) the scalar product of the eigenfunction (2.17) and dual eigenfunction (2.20) is equal to

$$
\begin{gathered}
\left\langle 0\left|C\left(\lambda_{1}\right) \ldots C\left(\lambda_{N}\right) B\left(\lambda_{1}\right) \ldots B\left(\lambda_{N}\right)\right| 0\right\rangle=(\sin 2 \eta)^{N}\left\{\prod_{j=1}^{N} a\left(\lambda_{j}\right) d\left(\lambda_{j}\right)\right\} \\
\cdot\left\{\prod_{j=1}^{N} \prod_{\substack{K=1 \\
j \neq K}}^{N} f\left(\lambda_{j}, \lambda_{K}\right)\right\}\langle 0 \mid 0\rangle \operatorname{det}_{N}\left\{\partial \varphi_{K} / \partial \lambda_{j}\right\} .
\end{gathered}
$$

Here the $\lambda_{j}$ satisfy the system of transcendental equations (2.18), (2.5). The Jacobi matrix can be written down in the explicit form

$$
\begin{aligned}
\frac{\partial \varphi_{K}}{\partial \lambda_{j}}= & \delta_{K j}\left\{i \frac{d}{d \lambda_{K}} \ln \frac{a\left(\lambda_{K}\right)}{d\left(\lambda_{K}\right)}+\sum_{l=1}^{N} \frac{\sin 4 \eta}{\sinh \left(\lambda_{K}-\lambda_{l}+2 i \eta\right) \sinh \left(\lambda_{K}-\lambda_{l}-2 i \eta\right)}\right\} \\
& -\frac{\sin 4 \eta}{\sinh \left(\lambda_{K}-\lambda_{j}+2 i \eta\right) \sinh \left(\lambda_{K}-\lambda_{j}-2 i \eta\right)}
\end{aligned}
$$

It would seem that (4.1) has pole whenever two $\lambda_{j}$ coincide $\left(\lambda_{1}=\lambda_{2}\right)$. Remember that this can hardly ever happen owing to the addition of the new Eq. (2.22) to the system (2.18). But if, by chance, this system has a solution, the limit of (4.1) is finite. Indeed, formally (4.1) has a pole of second order as $\lambda_{1} \rightarrow \lambda_{2}$. So the structure of the singular part is as follows

$$
a_{2} \cdot\left(\lambda_{1}-\lambda_{2}\right)^{-2}+a_{1} \cdot\left(\lambda_{1}-\lambda_{2}\right)^{-1} .
$$

One can show that $a_{2}$ is proportional to the left hand side of (2.22) and therefore $a_{2}=0$. While $a_{1}$ is zero owing to the symmetry of (4.1) under the permutation $\lambda_{1} \rightarrow \lambda_{2}$ (see the next section). The whole paper is devoted to the derivation of formula (4.1). 
Let us calculate the norms for the $X X Z$ model. The Hamiltonian of the model is

$$
H=\sum_{K=1}^{M}\left[\sigma_{K}^{1} \sigma_{K+1}^{1}+\sigma_{K}^{2} \sigma_{K+1}^{2}+\cos 2 \eta\left(\sigma_{K}^{3} \sigma_{K+1}^{3}-1\right)\right] .
$$

This model was treated by means of QISM in [7]. Its monodromy matrix is given by

$$
T(\lambda)=L_{M}(\lambda) \ldots L_{1}(\lambda)
$$

Her $L_{K}$ is the weight matrix for the 6 vertex model

$$
L_{K}(\lambda)=\left|\begin{array}{cc}
\sinh \left(\lambda-i \eta \sigma_{k}^{3}\right), & -i(\sin 2 \eta) \sigma_{K}^{-} \\
-i(\sin 2 \eta) \sigma_{K}^{+}, & \sinh \left(\lambda+i \eta \sigma_{K}^{3}\right)
\end{array}\right| .
$$

This monodromy matrix has the following property at real $\eta$ :

$$
T^{+}(\bar{\lambda})=\sigma^{2} T(\lambda) \sigma^{2}, \quad B^{+}(\bar{\lambda})=-C(\lambda) .
$$

Its communication relations is given by $R$ matrix (2.4), (2.5). The pseudovacuum is standard:

$$
|0\rangle=\prod_{j=1}^{M} \uparrow_{j}, \quad\langle 0|=| 0\rangle^{+}=\prod_{j=1}^{M}\left(\uparrow_{j}\right) .
$$

The notations $\uparrow_{j}$ and $\downarrow_{j}$ denotes vectors

$$
\uparrow=\left(\begin{array}{l}
1 \\
0
\end{array}\right), \quad \downarrow=\left(\begin{array}{l}
0 \\
1
\end{array}\right)
$$

at the $j$ site of the lattice. The vacuum eigenvalues are

$$
a(\lambda)=\sinh ^{M}(\lambda-i \eta), \quad d(\lambda)=\sinh ^{M}(\lambda+i \eta) .
$$

Properties (3.3), (3.4) are valid, at least in the limit $M \rightarrow \infty$. The trace identities look like

$$
H=-\left.2 i \sin 2 \eta \cdot \frac{\partial}{\partial \mu} \ln [A(\mu)+D(\mu)]\right|_{\mu=-i \eta}-2 M \cos 2 \eta .
$$

The eigenvalues of $H$ are

$$
4 \sum_{j=1}^{N}\left(\cos p_{j}-\cos 2 \eta\right)
$$

where

$$
\exp \{i p\}=\sinh (\lambda-i \eta) / \sinh (\lambda+i \eta)
$$

By means of (3.5), (4.1), (4.2) and (4.10) the square of the norm of the eigenfunction (2.17) can be calculated:

$$
\begin{aligned}
& \left\langle 0\left|B^{+}\left(\lambda_{1}\right) \ldots B^{+}\left(\lambda_{N}\right) B\left(\lambda_{1}\right) \ldots B\left(\lambda_{N}\right)\right| 0\right\rangle=(\sin 2 \eta)^{N} \\
& \cdot\left\{\prod_{j=1}^{N} \sinh ^{M}\left(\lambda_{j}-i \eta\right) \sinh ^{M}\left(\lambda_{j}+i \eta\right)\right\}\left\{\prod_{K>j}^{N} \frac{\sinh \left(\lambda_{j}-\lambda_{K}+2 i \eta\right) \sinh \left(\lambda_{j}-\lambda_{K}-2 i \eta\right)}{\sinh ^{2}\left(\lambda_{j}-\lambda_{K}\right)}\right\} \\
& \cdot \operatorname{det}_{N}\left\{\delta_{K j}\left[M \cdot \chi\left(\lambda_{K}, \eta\right)-\sum_{l=1}^{N} \chi\left(\lambda_{K}-\lambda_{l}, 2 \eta\right)\right]+\chi\left(\lambda_{K}-\lambda_{j}, 2 \eta\right)\right\} .
\end{aligned}
$$


Here the function of two variables $\chi(\lambda, \eta)$ is defined as follows:

$$
\chi(\lambda, \eta)=\sin 2 \eta / \sinh (\lambda-i \eta) \sinh (\lambda+i \eta) .
$$

Formula (4.14) for the norm of the coordinate wave function was presented in [9] and explicitly verified by direct calculations for $N=2,3$. Notice that the norm formula for the $X X X$ model may be obtained from (4.14) in the limit (2.7). In the papers [17], [18] the lattice quantum sine-Gordon model was constructed. The norms in this model can also be calculated by means of (4.1).

\section{Properties of the Jacobian}

Our aim is to prove (4.1). We shall see that it is valid even if (3.3)-(3.5) are not valid. Let us introduce the value

$$
\left\|\lambda_{1} \ldots \lambda_{N}\right\|_{N}=\frac{\left\langle 0\left|C\left(\lambda_{1}\right) \ldots C\left(\lambda_{N}\right) B\left(\lambda_{1}\right) \ldots B\left(\lambda_{N}\right)\right| 0\right\rangle}{(\sin 2 \eta)^{N}\left\{\prod_{j=1}^{N} a\left(\lambda_{j}\right) d\left(\lambda_{j}\right)\right\}\left\{\prod_{\substack{j \neq K \\ 1}}^{N} f\left(\lambda_{j}, \lambda_{K}\right)\right\}\langle 0 \mid 0\rangle},
$$

which is proportional to the scalar product of the eigenfunction (2.17) and dual eigenfunction (2.20). In (5.1) the values $\lambda_{j}$ satisfy the system (2.18). The formula (4.1) is equivalent to

$$
\left\|\lambda_{1} \ldots \lambda_{N}\right\|_{N}=\operatorname{det}_{N}\left\{\partial \varphi_{K} / \partial \lambda_{j}\right\}
$$

During the proof we shall put $\langle 0 \mid 0\rangle=1$.

Let us first of all study the properties of the Jacobian on the right hand side of (5.2). The most important ones are as follows. The Jacobian is a symmetric function of all $\lambda_{j}$. For the proof it is sufficient to interchange the position of two lines and two columns.

Let us remind the reader that the vacuum eigenvalues $a(\lambda)$ and $d(\lambda)$ are arbitrary functions. This permits us to consider the values

$$
X_{p}=i \frac{d}{d \lambda_{p}} \ln \left(\frac{a\left(\lambda_{p}\right)}{d\left(\lambda_{p}\right)}\right)
$$

as variables independent of $\lambda_{j}$, see Appendix B. These variables can change freely at fixed $\lambda_{j}$. We shall study the dependence of the Jacobian on $X_{p}$. Let us reformulate the symmetry property. The Jacobian is invariant under simultaneous replacement $\lambda_{K} \leftrightarrow \lambda_{j}$ and $X_{K} \leftrightarrow X_{j}$. Due to (4.2) the Jacobian is a linear function in $X_{1}$

$$
\operatorname{det}_{N}\left\{\partial \varphi_{K} / \partial \lambda_{j}\right\}=U_{1} X_{1}+V_{1} .
$$

Values $V_{1}$ and $U_{1}$ are $X_{1}$-independent. They depend on all other $X_{p}, P=2, \ldots, N$ and on all $\lambda_{j}$. Due to the symmetry the Jacobian is a linear function in each $X_{p}$. The coefficient $U_{1}$ in (5.4) is equal to another Jacobian

$$
\begin{gathered}
U_{1}=\operatorname{det}_{N-1}\left\{\partial \hat{\varphi}_{K} / \partial \lambda_{j}\right\}, \quad K, j=2, \ldots, N, \\
\hat{\varphi}_{K}=i \ln \frac{\hat{a}\left(\lambda_{K}\right)}{\hat{d}\left(\lambda_{K}\right)}+i \sum_{\substack{j=2 \\
j \neq K}}^{N} \ln \frac{f\left(\lambda_{K}, \lambda_{j}\right)}{f\left(\lambda_{j}, \lambda_{K}\right)}, \\
\hat{a}(\lambda)=a(\lambda) \cdot f\left(\lambda, \lambda_{1}\right), \quad \hat{d}(\lambda)=d(\lambda) f\left(\lambda_{1}, \lambda\right) .
\end{gathered}
$$


So $U_{1}$ differs from the initial Jacobian by removing the first line and first column and by replacing the vacuum eigenvalues $a(\lambda)$ and $d(\lambda)$ by $\hat{a}(\lambda)$ and $\hat{d}(\lambda)$ [see (5.7)]. This is a direct consequence of (4.2). Notice that in (5.6) $\lambda_{j}, j=2, \ldots, N$ are solutions of a reduced system of TE

$$
\left[\hat{a}\left(\lambda_{n}\right) / \hat{d}\left(\lambda_{n}\right)\right] \prod_{\substack{j=2 \\ j \neq n}}^{N}\left[f\left(\lambda_{n}, \lambda_{j}\right) / f\left(\lambda_{j}, \lambda_{n}\right)\right]=1, n=2, \ldots, N .
$$

The Jacobian is equal to zero at all $X_{p}=0$ and $\lambda_{j}$ fixed:

$$
\left.\operatorname{det}_{N}\left\{\partial \varphi_{K} / \partial \lambda_{j}\right\}\right|_{X_{p}=0}=0, \quad p=1, \ldots, N .
$$

Indeed

$$
\left.\operatorname{det}_{N}\left\{\partial \varphi / \partial \lambda_{j}\right\}\right|_{X=0}=\operatorname{det} M_{j K},
$$

and by means of (4.2) and (4.15) we have

$$
M_{j K}=\delta_{K j}\left\{\sum_{l=1}^{N} \chi\left(\lambda_{K}-\lambda_{l}, 2 \eta\right)\right\}-\chi\left(\lambda_{K}-\lambda_{j}, 2 \eta\right) .
$$

This matrix has an eigenvector with zero eigenvalue:

$$
\sum_{K=1}^{N} M_{j K} V_{K}=0, \quad V_{K}=1 .
$$

So $\operatorname{det} M=0$ and (5.9) is proved.

At last

$$
\operatorname{det}_{1}\left\{\partial \varphi_{j} / \partial \lambda_{K}\right\}=X_{1}=i \frac{d}{d \lambda_{1}} \ln \frac{a\left(\lambda_{1}\right)}{d\left(\lambda_{1}\right)} .
$$

It is clear that $\left\|\lambda_{1} \ldots \lambda_{N}\right\|_{N}$, see (5.1), must possess the same properties. We shall prove the following.

Theorem. To prove formula (5.2) it is sufficient to prove that $\left\|\lambda_{1} \ldots \lambda_{N}\right\|_{N}$ has the following properties.

1) It is invariant under simultaneous replacement

$$
\lambda_{K} \leftrightarrow \lambda_{j} \text { and } X_{K} \leftrightarrow X_{j} .
$$

2) It is a linear function in $X_{1}(5.3)$

$$
\left\|\lambda_{1}, \ldots, \lambda_{N}\right\|_{N}=U_{1} \cdot X_{1}+V_{1} .
$$

3) The coefficient $U_{1}$ is equal to

$$
U_{1}=\left\|\lambda_{2}, \ldots, \lambda_{N}\right\|_{N-1}^{\bmod } .
$$

It is given by formula (5.1) in the case of $N-1$ particles. The particle corresponding to $\lambda_{1}$ is removed and $a(\lambda)$ and $d(\lambda)$ are replaced by $\hat{a}(\lambda)$ and $\hat{d}(\lambda)$ [see (5.7)].

4) It is equal to zero at all $X_{p}=0$ and $\lambda_{j}$ fixed

$$
\left\|\lambda_{1}, \ldots, \lambda_{N}\right\|_{N}=0 \text { and } X_{p}=0, \quad p=1, \ldots, N .
$$


5) For the one particle state

$$
\left\|\lambda_{1}\right\|_{1}=X_{1} \text {. }
$$

The proof goes by induction in the number of particles starting from $N=1$ [compare (5.10) and (5.15)]. Assume that (5.2) is already proved for $N=1, \ldots, q-1$. We shall show that it is valid for $N=q$. Let us consider the value

$$
\Delta_{q}=\left\|\lambda_{1}, \ldots, \lambda_{q}\right\|_{q}-\operatorname{det}_{q}\left(\partial \varphi_{K} / \partial \lambda_{j}\right) .
$$

It is a linear function in $X_{1}$, see (5.4), (5.12). The derivative with respect to $X_{1}$ is equal to

$$
\partial \Delta_{q} / \partial X_{1}=\left\|\lambda_{2}, \ldots, \lambda_{q}\right\|_{q-1}^{\bmod }-\operatorname{det}_{q-1}\left\{\partial \varphi_{K} / \partial \lambda_{j}\right\},
$$

see (5.5), (5.13). The right hand side is equal to zero due to the inductive assumption. So $\partial \Delta_{q} / \partial X_{1}=0$ and $\Delta_{q}$ is $X_{1}$ independent. Due to the symmetry (5.11) $\Delta_{q}$ does not depend on any of $X_{p}$. But due to (5.9) and (5.14).

$$
\Delta_{q}=0 \quad \text { at } \quad X_{p}=0, \quad p=1, \ldots, q .
$$

So $\Delta_{q}=0$ identically. So (5.2) is valid for $N=q$, which concludes the proof of the theorem. In the remaining part of the paper we shall prove that $\left\|\lambda_{1} \ldots \lambda_{N}\right\|[$ see (5.1)] possesses the five properties mentioned in the theorem.

\section{Description of the Scalar Products}

We begin with the study of the scalar products in the generic case

$$
\left\langle 0\left|C\left(\lambda_{1}^{C}\right) \ldots C\left(\lambda_{N}^{C}\right) B\left(\lambda_{1}^{B}\right) \ldots B\left(\lambda_{N}^{B}\right)\right| 0\right\rangle .
$$

All the $\lambda_{j}^{C}$ and $\lambda_{K}^{B}$ are assumed to be different and independent; in general they do not satisfy any equation. The left hand side of (4.1) is a particular case of (6.1). We shall deal only with the $R$ matrix (2.8). By means of (2.9)-(2.16) one can calculate (6.1) completely. For example,

$$
\left\langle 0\left|C\left(\lambda^{C}\right) B\left(\lambda^{B}\right)\right| 0\right\rangle=g\left(\lambda^{C}, \lambda^{B}\right)\left\{a\left(\lambda^{C}\right) d\left(\lambda^{B}\right)-a\left(\lambda^{B}\right) d\left(\lambda^{C}\right)\right\} .
$$

Let us try to characterize (6.1) for any $N$. Notice that after the calculation each $\lambda_{j}^{B}$ and $\lambda_{K}^{C}$ must become either the argument of $a(\lambda)$ or of $d(\lambda)$. In this way each term in the expression for (6.1) corresponds to some partition of the set

$$
S_{N} \equiv\left\{\lambda_{1}^{C}, \ldots, \lambda_{N}^{C}, \lambda_{1}^{B}, \ldots, \lambda_{N}^{B}\right\}=\left\{\lambda^{C}\right\} \cup\left\{\lambda^{B}\right\}
$$

into two disjoint subsets $\left\{\lambda^{A}\right\}$ and $\left\{\lambda^{D}\right\}$ :

$$
\left\{\lambda^{A}\right\} \cap\left\{\lambda^{D}\right\}=\emptyset, \quad\left\{\lambda^{A}\right\} \cup\left\{\lambda^{D}\right\}=S_{N} .
$$

Let us denote the number of elements in the subset $\left\{\lambda^{A}\right\}$ by $\operatorname{card} A$ and for $\left\{\lambda^{D}\right\} \operatorname{card} D(\operatorname{card} A+\operatorname{card} D=2 N)$. So we have:

$$
\begin{aligned}
& \left\langle 0\left|C\left(\lambda_{1}^{C}\right) \ldots C\left(\lambda_{N}^{C}\right) B\left(\lambda_{1}^{B}\right) \ldots B\left(\lambda_{N}^{B}\right)\right| 0\right\rangle \\
& \quad=\sum_{\text {partitions }} K_{N}\left(\begin{array}{ll}
\left\{\lambda^{C}\right\} & \left\{\lambda^{B}\right\} \\
\left\{\lambda^{A}\right\} & \left\{\lambda^{D}\right\}
\end{array}\right) \prod_{j=1}^{\operatorname{card} A} a\left(\lambda_{j}^{A}\right) \prod_{K=1}^{\operatorname{card} D} d\left(\lambda_{K}^{D}\right) .
\end{aligned}
$$


The coefficients $K_{N}$ are some explicit functions of all the $\lambda$, constructed by means of $f$ and $g$ (2.5), independent of vacuum eigenvalues. We claim that the only nonzero elements in (6.5) are those for which

$$
\operatorname{card} A=\operatorname{card} D=N \text {. }
$$

Indeed let us apply formula (6.5) for the monodromy matrix (2.25), depending on arbitrary $y \in C$. The left hand side of (6.5) is manifestly $y$ independent, while on the right hand side each term is multiplied by the factor $\exp \{\operatorname{card} A-\operatorname{card} D) \cdot \ln y\}$. So the $y$ independent terms are characterized by (6.6). All other terms must vanish. Let us rewrite (6.5):

$$
\left\langle 0\left|\prod_{j=1}^{N} C\left(\lambda_{j}^{C}\right) \prod_{K=1}^{N} B\left(\lambda_{K}^{B}\right)\right| 0\right\rangle=\sum_{\text {partitions }}\left\{\prod_{j=1}^{N} a\left(\lambda_{j}^{A}\right) d\left(\lambda_{j}^{D}\right)\right\} K_{N}\left(\begin{array}{ll}
\left\{\lambda^{C}\right\} & \left\{\lambda^{B}\right\} \\
\left\{\lambda^{A}\right\} & \left\{\lambda^{D}\right\}
\end{array}\right) .
$$

The number of terms in the right hand side is equal to $C_{2 N}^{N}$. In the explicit expression for $K$ the following intersections are essential:

$$
\begin{aligned}
& \left\{\lambda^{A}\right\} \cap\left\{\lambda^{C}\right\}=\left\{\lambda^{A C}\right\}, \quad\left\{\lambda^{D}\right\} \cap\left\{\lambda^{C}\right\}=\left\{\lambda^{D C}\right\} \\
& \left\{\lambda^{A}\right\} \cap\left\{\lambda^{B}\right\}=\left\{\lambda^{A B}\right\}, \quad\left\{\lambda^{D}\right\} \cap\left\{\lambda^{B}\right\}=\left\{\lambda^{D B}\right\} .
\end{aligned}
$$

Obviously one has following trivial identities:

$$
\begin{array}{ll}
\left\{\lambda^{A C}\right\} \cup\left\{\lambda^{A B}\right\}=\left\{\lambda^{A}\right\}, & \left\{\lambda^{A C}\right\} \cup\left\{\lambda^{D C}\right\}=\left\{\lambda^{C}\right\} \\
\left\{\lambda^{D C}\right\} \cup\left\{\lambda^{D B}\right\}=\left\{\lambda^{D}\right\}, \quad\left\{\lambda^{A B}\right\} \cup\left\{\lambda^{D B}\right\}=\left\{\lambda^{B}\right\} .
\end{array}
$$

The number of elements in the subsets (6.8) satisfy the following identities:

$$
n=\operatorname{card}(D C)=\operatorname{card}(A B), \quad \operatorname{card}(A C)=\operatorname{card}(D B)=N-n .
$$

An example of the partition in (6.7) is

$$
\left\{\lambda^{A}\right\}=\left\{\lambda^{C}\right\}, \quad\left\{\lambda^{D}\right\}=\left\{\lambda^{B}\right\}
$$

In this case we have

$$
\left\{\lambda^{A C}\right\}=\left\{\lambda^{C}\right\}, \quad\left\{\lambda^{A B}\right\}=\emptyset, \quad\left\{\lambda^{D C}\right\}=\emptyset, \quad\left\{\lambda^{D B}\right\}=\left\{\lambda^{B}\right\}, \quad n=0 .
$$

We shall call the term in (6.7) corresponding to this partition the highest term. In Sect. 8 we shall demonstrate the following properties of $K_{N}$. First of all the highest $K$ is equal to

$$
K_{N}\left(\begin{array}{ll}
\left\{\lambda^{C}\right\} & \left\{\lambda^{B}\right\} \\
\left\{\lambda^{C}\right\} & \left\{\lambda^{B}\right\}
\end{array}\right)=Q_{N}\left(\left\{\lambda_{l}^{B}\right\},\left\{\lambda_{p}^{C}\right\}\right)\left\{\prod_{j=1}^{N} \prod_{K=1}^{N} \sinh ^{-1}\left(\lambda_{K}^{B}-\lambda_{j}^{C}\right)\right\} .
$$

Here $Q_{N}$ is a function of $2 N$ variables, which is symmetric separately in all $\lambda_{j}^{B}$ and in all $\lambda_{K^{C}}^{C} . Q_{N}$ depends on an individual $\lambda_{j}^{B}$ for fixed values of the remaining variables as follows:

$$
Q_{N}\left(\left\{\lambda^{B}\right\},\left\{\lambda^{C}\right\}\right)=e^{-(N-1) \lambda_{j}^{B}} \cdot P_{N-1}\left(e^{2 \lambda_{j}^{B}}\right) .
$$

Here $P_{N-1}$ is a polynomial of degree $(N-1)$, with coefficients depending on all other variables. Similarly $Q_{N}$ depends on individual $\lambda_{K}^{C}$ as follows:

$$
Q_{N}\left(\left\{\lambda^{B}\right\},\left\{\lambda^{C}\right\}\right)=e^{(N-1) \lambda_{\mathrm{K}}^{C}} \tilde{P}_{N-1}\left(e^{-2 \lambda_{\mathrm{K}}^{C}}\right),
$$


where $\tilde{P}_{N-1}$ is another polynomial of degree $(N-1)$. The most remarkable property is the following. If one of the $\lambda^{B}$ coincides with one of $\lambda^{C}$, say $\lambda_{1}^{B}=\lambda_{1}^{C}$, our $Q_{N}$ is expressed in terms of $Q_{N-1}$ with $\lambda_{1}^{B}$ and $\lambda_{1}^{C}$ removed

$$
\begin{aligned}
\left.Q_{N}\left(\left\{\lambda_{l}^{B}\right\},\left\{\lambda_{j}^{C}\right\}\right)\right|_{\lambda_{1}^{C}=\lambda_{1}^{B}}= & (-i \sin 2 \eta)\left\{\prod_{K=2}^{N} \sinh \left(\lambda_{1}^{B}-\lambda_{K}^{C}-2 i \eta\right)\right\} \\
& \cdot\left\{\prod_{j=2}^{N} \sinh \left(\lambda_{j}^{B}-\lambda_{1}^{C}-2 i \eta\right)\right\} Q_{N-1}\left(\left\{\lambda_{l \neq 1}^{B}\right\},\left\{\lambda_{K \neq 1}^{C}\right\}\right) .
\end{aligned}
$$

Notice that $Q_{1}$ has already been calculated in (6.2)

$$
Q_{1}=-i \sin 2 \eta
$$

Due to the symmetry of $Q_{N}$ it can be reduced to $Q_{N-1}$ in all points $\lambda_{K}^{C}=\lambda_{j}^{B}$. One can show that all these properties fix $Q_{N}$ in a unique way.

An arbitrary coefficient in (6.7) is expressed as follows :

$$
K_{N}\left(\begin{array}{ll}
\left\{\lambda^{C}\right\} & \left\{\lambda^{B}\right\} \\
\left\{\lambda^{A}\right\} & \left\{\lambda^{D}\right\}
\end{array}\right)=\pi_{N}\left(\begin{array}{ll}
\left\{\lambda^{C}\right\} & \left\{\lambda^{B}\right\} \\
\left\{\lambda^{A}\right\} & \left\{\lambda^{D}\right\}
\end{array}\right)\left\{\prod_{j=1}^{N} \prod_{K=1}^{N} \sinh ^{-1}\left(\lambda_{K}^{D}-\lambda_{j}^{A}\right)\right\} .
$$

Here the Laurent polynomial $\pi_{N}$ is equal to

$$
\begin{aligned}
\pi_{N}\left(\begin{array}{ll}
\left\{\lambda^{C}\right\} & \left\{\lambda^{B}\right\} \\
\left\{\lambda^{A}\right\} & \left\{\lambda^{D}\right\}
\end{array}\right)=Q_{N-n}\left(\left\{\lambda^{D B}\right\},\left\{\lambda^{A C}\right\}\right) Q_{n}\left(\left\{\lambda^{D C}\right\},\left\{\lambda^{A B}\right\}\right) \\
\cdot\left\{\prod_{j \in D C} \prod_{K \in A C} \sinh \left(\lambda_{j}^{D C}-\lambda_{K}^{A C}-2 i \eta\right)\right\}\left\{\prod_{j \in D B} \prod_{K \in A B} \sinh \left(\lambda_{j}^{D B}-\lambda_{K}^{A B}-2 i \eta\right)\right\} .
\end{aligned}
$$

For $n$ see (6.10). The double product in the third factor on the right hand side means that $\lambda_{j}^{D C}$ runs through all the set $\left\{\lambda^{D C}\right\}$, and $\lambda_{K}^{A C}$ in an independent way runs through all the set $\left\{\lambda^{A C}\right\}$. The last factor has a similar meaning. So we have completed the full description of the scalar products. We shall prove these formulas as follows. In the next section we shall construct a special case of the 6 vertex model with a lot of free parameters. We shall apply formula (6.7) for this model and choose parameters in such a way that all terms, except one, on the right hand side will be equal to zero. This will allow us to investigate the properties of an individual coefficient $K_{N}$. Notice that scalar products for the $X X Z$ model itself were studied in [10].

\section{Special Case of the 6 Vertex Model}

We shall consider the inhomogeneous generalization of the monodromy matrix (4.5). Namely we assume the spectral parameter in the $L$ matrix (4.6) to be site dependent

$$
\begin{aligned}
L_{K}\left(\lambda-v_{K}\right)= & \begin{array}{cc}
\sinh \left(\lambda-v_{K}-i \eta \sigma_{K}^{3}\right), & -i \sigma_{K}^{-} \sin 2 \eta \\
-i \sigma_{K}^{+} \sin 2 \eta, & \sinh \left(\lambda-v_{K}+i \eta \sigma_{K}^{3}\right)
\end{array} \mid \\
= & \cos \eta \cdot \sinh \left(\lambda-v_{K}\right)-i \sigma^{3} \cdot \sigma_{K}^{3} \cdot \sin \eta \cdot \cosh \left(\lambda-v_{K}\right) \\
& -i \sin 2 \eta\left(\sigma^{-} \cdot \sigma_{K}^{+}+\sigma^{+} \cdot \sigma_{K}^{-}\right) .
\end{aligned}
$$


The monodromy matrix

$$
T(\lambda)=L_{M}\left(\lambda-v_{M}\right) \ldots L_{1}\left(\lambda-v_{1}\right)
$$

obeys the commutation relations (2.3) with the $R$ matrix (2.8). The pseudovacuum is the old one, see (4.8). The vacuum eigenvalues are now equal to

$$
a(\lambda)=\prod_{j=1}^{M} \sinh \left(\lambda-v_{j}-i \eta\right), \quad d(\lambda)=\prod_{j=1}^{M} \sinh \left(\lambda-v_{j}+i \eta\right) .
$$

Such an inhomogeneous generalization was used for example in [19-21].

Let us consider the special state

$$
B\left(\lambda_{M}\right) \ldots B\left(\lambda_{1}\right)|0\rangle \text {. }
$$

Here the number of the particles [of the $B(\lambda)$ ] is equal to the number of the sites in the lattice $N=M$. Below we shall write $N$ instead of $M$. All $\lambda_{j}$ in (7.4) are free. In Appendix $C$ it is shown that in the state (7.4) all spins are overturned, see (C.9),

$$
B\left(\lambda_{N}\right) \ldots B\left(\lambda_{1}\right)|0\rangle=\Omega \cdot Z_{N} .
$$

Here $Z_{N}$ is a $c$-number and

$$
\Omega=\prod_{j=1}^{N}\left(\downarrow_{j}\right)
$$

The definition of $Z_{N}$ is

$$
Z_{N}\left(\left\{\lambda_{\alpha}\right\},\left\{v_{K}\right\}\right)=\Omega \prod_{j=1}^{N} B\left(\lambda_{\alpha}\right) \cdot|0\rangle .
$$

Several representations for $Z_{N}$ are given in Appendix D. Let us study the properties of the $Z_{N}$. Due to (2.10) $Z_{N}$ is symmetric in all $\lambda_{\alpha}$. $Z_{N}$ depends on individual $\lambda_{\alpha}$ as follows (all other variables kept fixed):

$$
Z_{N}=\exp \left\{-(N-1) \lambda_{\alpha}\right\} P_{N-1}\left(e^{2 \lambda_{\alpha}}\right) .
$$

Here $P_{N-1}$ is a polynomial of degree $N-1$, with coefficients dependent on all other variables. Indeed, one can see by inspection that $B(\lambda)$ depends on $\lambda$ like (7.8). From representation (D.21) one can see that $Z_{N}$ depends on $v_{j}$ in an anlogous fashion. $Z_{N}$ is symmetric in all $v_{j}$. $Z_{N}$ depends on an individual $v_{j}$ as follows:

$$
Z_{N}=\exp \left\{(N-1) v_{j}\right\} \tilde{P}_{N-1}\left(e^{-2 v_{j}}\right) .
$$

Here $\tilde{P}_{N-1}$ is another polynomial of degree $N-1$. Formula (D.24) shows that if

$$
v_{1}=\lambda_{1}+i \eta
$$

then $Z_{N}$ can be expressed in terms of $Z_{N-1}$ with $v_{1}$ and $\lambda_{1}$ removed:

$$
\begin{aligned}
\left.Z_{N}\left(\left\{\lambda_{\alpha}\right\},\left\{v_{j}\right\}\right)\right|_{v_{1}-\lambda_{1}=i \eta}= & -i \sin 2 \eta\left\{\prod_{K=2}^{N} \sinh \left(\lambda_{1}-v_{K}-i \eta\right)\right\} \\
& \cdot\left\{\prod_{\alpha=2}^{N} \sinh \left(\lambda_{\alpha}-v_{1}-i \eta\right)\right\} \cdot Z_{N-1}\left(\left\{\lambda_{\alpha \neq 1}\right\},\left\{v_{j \neq 1}\right\}\right) .
\end{aligned}
$$


Due to the symmetry of $Z_{N}$, similar formulas are valid in all points $v_{X}=i \eta+\lambda_{\beta}$. For $N=1$

$$
Z_{1}=-i \sin 2 \eta
$$

In the next section we shall use this model to study the coefficients in the formula (6.7). Notice that the norm of the highest eigenvector of the transfer matrix (7.2) was calculated for the very special set of $v_{K}$ in $[10]$.

\section{Proof of the Formula for the Scalar Product}

Let us calculate the scalar product of the state (7.4) and the dual state

$$
\langle 0| C\left(\lambda_{1}^{C}\right) \ldots C\left(\lambda_{N}^{C}\right), \quad N=M
$$

in the inhomogeneous 6 vertex model by means of formula (6.7). We shall try to choose the free parameters $v_{j}$ to annihilate all terms on the right hand side of (6.7) except one. Put e.g.

$$
v_{j}=\lambda_{j}^{C}+i \eta
$$

Now the vacuum eigenvalues (7.3) are equal to

$$
a(\lambda)=\prod_{j=1}^{N} \sinh \left(\lambda-\lambda_{j}^{c}-2 i \eta\right), \quad d(\lambda)=\prod_{j=1}^{N} \sinh \left(\lambda-\lambda_{j}^{c}\right) .
$$

As $d\left(\lambda_{K}^{C}\right)=0$ all $\lambda_{j}^{C}$ must become arguments of $a(\lambda)$. So on the right hand side of $(6.7)$ only the highest term remains:

$$
\begin{aligned}
& \left\langle 0\left|\prod_{j=1}^{N} C\left(\lambda_{j}^{C}\right) \prod_{l=1}^{N} B\left(\lambda_{l}^{B}\right)\right| 0\right\rangle \\
& \quad=K_{N}\left(\begin{array}{ll}
\left\{\lambda^{C}\right\} & \left\{\lambda^{B}\right\} \\
\left\{\lambda^{C}\right\} & \left\{\lambda^{B}\right\}
\end{array}\right) \times \prod_{j=1}^{N} \prod_{K=1}^{N} \sinh \left(\lambda_{K}^{B}-\lambda_{j}^{C}\right) \cdot \sinh \left(\lambda_{K}^{C}-\lambda_{j}^{C}-2 i \eta\right) .
\end{aligned}
$$

Let us calculate the left hand side

$$
\begin{aligned}
\left\langle 0\left|\prod_{j=1}^{N} C\left(\lambda_{j}^{C}\right) \prod_{l=1}^{N} B\left(\lambda_{l}^{B}\right)\right| 0\right\rangle & =\left\{\langle 0| \prod_{j=1}^{N} C\left(\lambda_{j}^{C}\right) \Omega\right\}\left\{\Omega \prod_{j=1}^{N} B\left(\lambda_{j}^{B}\right)|0\rangle\right\} \\
& =Z_{N}\left(\left\{\lambda_{j}^{C}\right\},\left\{\lambda_{j}^{C}+i \eta\right\}\right) Z_{N}\left(\left\{\lambda_{l}^{B}\right\},\left\{\lambda_{j}^{C}+i \eta\right\}\right) .
\end{aligned}
$$

Here we have used the formulas (7.5), (7.7) and (D.17). The first factor on the right hand side of (8.5) can be calculated by means of (7.11)

$$
Z_{N}\left(\left\{\lambda_{j}^{C}\right\},\left\{\lambda_{j}^{C}+i \eta\right\}\right)=\prod_{K=1}^{N} \prod_{j=1}^{N} \sinh \left(\lambda_{K}^{C}-\lambda_{j}^{C}-2 i \eta\right) .
$$

Combining (8.4)-(8.6), we obtain

$$
K_{N}\left(\begin{array}{ll}
\left\{\lambda^{C}\right\} & \left\{\lambda^{B}\right\} \\
\left\{\lambda^{C}\right\} & \left\{\lambda^{B}\right\}
\end{array}\right)=\left\{\prod_{j=1}^{N} \prod_{K=1}^{N} \sinh ^{-1}\left(\lambda_{K}^{B}-\lambda_{j}^{C}\right)\right\} Z_{N}\left(\left\{\lambda_{l}^{B}\right\},\left\{\lambda_{j}^{C}+i \eta\right\}\right) .
$$

If we change the notations

$$
Q_{N}\left(\left\{\lambda_{l}^{B}\right\},\left\{\lambda_{j}^{C}\right\}\right) \equiv Z_{N}\left(\left\{\lambda_{j}^{B}\right\},\left\{\lambda_{l}^{C}+i \eta\right\}\right),
$$


then it will be obvious that (8.7) and (6.13) coincide. Also properties (7.8)-(7.12) turn into properties (6.14)-(6.17). In this way we have proved all the properties of the highest term and of $Q_{N}$.

Let us discuss now an arbitrary coefficient in (6.7). We fix the partition corresponding to one of the terms in (6.7) $\left\{\lambda^{A}\right\},\left\{\lambda^{D}\right\}$ and choose

$$
v_{j}=\lambda_{j}^{A}+i \eta \text {. }
$$

This leads to

$$
a(\lambda)=\prod_{j=1}^{N} \sinh \left(\lambda-\lambda_{j}^{A}-2 i \eta\right), \quad d(\lambda)=\prod_{j=1}^{N} \sinh \left(\lambda-\lambda_{j}^{A}\right),
$$

and so $d\left(\lambda_{j}^{A}\right)=0$. All the $\lambda_{j}^{A}$ must become the arguments of $a(\lambda)$. Therefore the only nonzero term on the right hand side of (6.7) just corresponds to the fixed partition

$$
\begin{aligned}
\left\langle 0\left|\prod_{j=1}^{N} C\left(\lambda_{j}^{C}\right) \prod_{l=1}^{N} B\left(\lambda^{B}\right)\right| 0\right\rangle= & \left\{\prod_{K=1}^{N} \prod_{j=1}^{N} \sinh \left(\lambda_{K}^{A}-\lambda_{j}^{A}-2 i \eta\right)\right\} \\
& \cdot\left\{\prod_{K=1}^{N} \prod_{j=1}^{N} \sinh \left(\lambda_{K}^{D}-\lambda_{j}^{A}\right)\right\} \cdot K_{N}\left(\begin{array}{ll}
\left\{\lambda^{C}\right\} & \left\{\lambda^{B}\right\} \\
\left\{\lambda^{A}\right\} & \left\{\lambda^{D}\right\}
\end{array}\right) .
\end{aligned}
$$

Let us calculate the left hand side

$$
\left\langle 0\left|\prod_{j=1}^{N} C\left(\lambda_{j}^{C}\right) \prod_{l=1}^{N} B\left(\lambda_{l}^{B}\right)\right| 0\right\rangle=Z_{N}\left(\left\{\lambda_{j}^{C}\right\},\left\{\lambda_{j}^{A}+i \eta\right\}\right) Z_{N}\left(\left\{\lambda_{l}^{B}\right\},\left\{\lambda_{j}^{A}+i \eta\right\}\right) .
$$

Since the intersections $\left\{\lambda^{A C}\right\}$ and $\left\{\lambda^{A B}\right\}$ are nonempty simultaneously, the right hand side of (8.12) can be transformed by means of (7.11). We obtain

$$
\begin{aligned}
\langle 0| & \prod_{j=1}^{N} C\left(\lambda_{j}^{C}\right) \prod_{l=1}^{N} B\left(\lambda_{l}^{B}\right)|0\rangle=Q_{n}\left(\left\{\lambda^{D C}\right\},\left\{\lambda^{A B}\right\}\right) Q_{N-n}\left(\left\{\lambda^{D B}\right\},\left\{\lambda^{A C}\right\}\right) \\
& \cdot\left\{\prod_{j=1}^{N} \prod_{K=1}^{N} \sinh \left(\lambda_{K}^{A}-\lambda_{j}^{A}-2 i \eta\right)\right\}\left\{\prod_{j \in D C} \prod_{K \in A C} \sinh \left(\lambda_{j}^{D C}-\lambda_{K}^{A C}-2 i \eta\right)\right\} \\
& \cdot\left\{\prod_{K \in D B} \prod_{j \in A B} \sinh \left(\lambda_{K}^{D B}-\lambda_{j}^{A B}-2 i \eta\right)\right\} .
\end{aligned}
$$

Here we use the notations (8.8), (6.8), (6.10). If we combine (8.11)-(8.13), we obtain (6.18), (6.19). In this way we finish the proof of all the formulas given in Sect. 6.

\section{Norm of an Arbitrary State}

Thus far we have described the general scalar product. To obtain the formula for the scalar product of the eigenfunction and its dual (4.1) we just do two steps. First of all we must let $\lambda_{j}^{C}$ tend to $\lambda_{j}^{B}$ in (6.7) in order to have the expression for the scalar product of an arbitrary wave function and its dual. Secondly we must let $\lambda_{j}^{C}=\lambda_{j}^{B}$ $=\lambda_{j}$ tend to a solution of the system of TE (2.18), (2.5). In this section we shall make the first step gradually. Let

$$
\lambda_{1}^{C} \rightarrow \lambda_{1}^{B}=\lambda_{1}
$$


while all the other $\lambda_{j}^{C}$ and $\lambda_{K}^{B}$ are fixed. We are interested in the dependence of the limit on the vacuum eigenvalues. There exist four types of terms on the right hand side of (6.7) which have different behavior in the limit (9.1).

1) Terms corresponding to partitions with the following properties $\lambda_{1}^{C} \in\left\{\lambda^{A}\right\}$, $\lambda_{1}^{B} \in\left\{\lambda^{A}\right\}$. These terms have finite limit and their dependence on the vacuum eigenvalues of argument $\lambda_{1}$ is the following: $a^{2}\left(\lambda_{1}\right)$.

2) Terms corresponding to the partitions $\lambda_{1}^{C} \in\left\{\lambda^{D}\right\}, \lambda_{1}^{B} \in\left\{\lambda^{D}\right\}$, whose dependence is $d^{2}\left(\lambda_{1}\right)$.

3) Terms corresponding to the partitions

$$
\lambda_{1}^{C} \in\left\{\lambda^{A}\right\}, \quad \lambda_{1}^{B} \in\left\{\lambda^{D}\right\} .
$$

We use following enumeration:

$$
\lambda_{1}^{C}=\lambda_{1}^{A}=\lambda_{1}^{A C}, \quad \lambda_{1}^{B}=\lambda_{1}^{D}=\lambda_{1}^{D B} .
$$

These terms have a pole in the limit (9.1) due to the factor

$$
\sinh ^{-1}\left(\lambda_{1}^{D}-\lambda_{1}^{A}\right)=\sinh ^{-1}\left(\lambda_{1}^{B}-\lambda_{1}^{C}\right)
$$

in the expression for $K_{N}$ (6.18). Let us calculate the residue. The terms of the third type are summed up to give

$$
a\left(\lambda_{1}^{C}\right) d\left(\lambda_{1}^{B}\right) \sum K_{N}\left(\begin{array}{ll}
\left\{\lambda_{1}^{C}, \lambda_{j}^{C}\right\} & \left\{\lambda_{1}^{B}, \lambda_{j}^{B}\right\} \\
\left\{\lambda_{1}^{C}, \lambda_{j}^{A}\right\} & \left\{\lambda_{1}^{B}, \lambda_{j}^{D}\right\}
\end{array}\right) \prod_{j=2}^{N} a\left(\lambda_{j}^{A}\right) d\left(\lambda_{j}^{D}\right) .
$$

Here $j=2, \ldots, N$ and the summation is carried out by partitions of

$$
S_{N-1}=\left\{\lambda_{j \neq 1}^{C}\right\} \cup\left\{\lambda_{j \neq 1}^{B}\right\}
$$

into two disjoint subsets $\left\{\lambda_{j \neq 1}^{A}\right\}$ and $\left\{\lambda_{j \neq 1}^{D}\right\}$, for which

$$
\operatorname{card} A=\operatorname{card} D=\operatorname{card} C=\operatorname{card} B=N-1
$$

[compare with (6.3), (6.4), (6.6), and (6.7)].

To calculate the residue we shall rewrite $K_{N}$ from (9.5) by means of (6.18)

$$
\begin{gathered}
K_{N}=\sinh ^{-1}\left(\lambda_{1}^{B}-\lambda_{1}^{C}\right)\left\{\prod_{K=2}^{N} \sinh ^{-1}\left(\lambda_{K}^{D}-\lambda_{1}^{C}\right)\right\} \cdot\left\{\prod_{j=2}^{N} \sinh ^{-1}\left(\lambda_{1}^{B}-\lambda_{j}^{A}\right)\right\} \\
\cdot\left\{\prod_{j=2}^{N} \prod_{K=2}^{N} \sinh ^{-1}\left(\lambda_{K}^{D}-\lambda_{j}^{A}\right)\right\} \pi_{N}\left|\left(\begin{array}{ll}
\left\{\lambda^{C}\right\} & \left\{\lambda^{B}\right\} \\
\left\{\lambda^{A}\right\} & \left\{\lambda^{D}\right\}
\end{array}\right)\right|_{\lambda_{1}^{A C} \rightarrow \lambda_{1}^{D B}} \cdot
\end{gathered}
$$

In Appendix $\mathrm{E}$ it is shown that if $\lambda_{1}^{A C}=\lambda_{1}^{D B}$, then $\pi_{N}$ is reduced to $\pi_{N-1}$ with $\lambda_{1}^{C}$ and $\lambda_{1}^{B}$ removed. Finally we have for the singular part of each $K_{N}$ in (9.5)

$$
K_{N} \rightarrow-i(\sin 2 \eta) \sinh ^{-1}\left(\lambda_{1}^{B}-\lambda_{1}^{C}\right)\left\{\prod_{K=2}^{N} f\left(\lambda_{K}^{A}, \lambda_{1}\right) f\left(\lambda_{1}, \lambda_{K}^{D}\right)\right\} K_{N-1}\left(\begin{array}{ll}
\left\{\lambda_{j \neq 1}^{C}\right\} & \left\{\lambda_{j \neq 1}^{B}\right\} \\
\left\{\lambda_{j \neq 1}^{A}\right\} & \left\{\lambda_{j \neq 1}^{D}\right\}
\end{array}\right) .
$$

Here we use the notation (2.5), (6.18) and formula (E.2). By means of (6.7) one can show that the singular part of the sum (9.5) is proportional to the scalar product 
for $N-1$ particles

$$
-i \sin 2 \eta \cdot \frac{a\left(\lambda_{1}^{C}\right) d\left(\lambda_{1}^{B}\right)}{\sinh \left(\lambda_{1}^{B}-\lambda_{1}^{C}\right)} \cdot\left\langle 0\left|\prod_{j=2}^{N} C\left(\lambda_{j}^{C}\right) \prod_{l=2}^{N} B\left(\lambda_{l}^{B}\right)\right| 0\right\rangle^{\mathrm{mod}} .
$$

Here "mod" means that during the calculation of this scalar product we must put

$$
\begin{array}{ll}
A(\lambda)|0\rangle=\hat{a}(\lambda)|0\rangle, & \hat{a}(\lambda)=a(\lambda) f\left(\lambda, \lambda_{1}\right), \\
D(\lambda)|0\rangle=\hat{d}(\lambda)|0\rangle, & \hat{d}(\lambda)=d(\lambda) f\left(\lambda_{1}, \lambda\right) .
\end{array}
$$

Before analyzing the finite part of these terms, let us study the terms of fourth type.

4) These terms correspond to the partitions

$$
\lambda_{1}^{C} \in\left\{\lambda^{D}\right\}, \quad \lambda_{1}^{B} \in\left\{\lambda^{A}\right\} .
$$

These terms have the same denominator (9.4), see (6.18). The singular part of the sum of these terms is calculated in just the same manner, by means of (E.5). This part is equal to

$$
i \sin 2 \eta \cdot \frac{a\left(\lambda_{1}^{B}\right) d\left(\lambda_{1}^{C}\right)}{\sinh \left(\lambda_{1}^{B}-\lambda_{1}^{C}\right)} \cdot\left\langle 0\left|\prod_{j=2}^{N} C\left(\lambda_{j}^{C}\right) \prod_{l=2}^{N} B\left(\lambda_{j}^{B}\right)\right| 0\right\rangle^{\bmod }
$$

and cancles (9.9) exactly.

Now we are in a position to calculate the finite part of terms 3) and 4). We must use l'Hôpital's rule. After calculating the indeterminacy one sees that the sum of 3) and 4) depends on the vacuum eigenvalue with argument $\lambda_{1}$ either as $a\left(\lambda_{1}\right) \cdot d\left(\lambda_{1}\right)$ or as $a^{\prime}\left(\lambda_{1}\right) d\left(\lambda_{1}\right), a\left(\lambda_{1}\right) d^{\prime}\left(\lambda_{1}\right)$. To calculate the coefficients of the last two terms is very simple. It is just the same as to calculate the residues (9.9), (9.12). The answer for the terms with derivatives is as follows:

$$
i \sin 2 \eta\left[a^{\prime}\left(\lambda_{1}\right) d\left(\lambda_{1}\right)-a\left(\lambda_{1}\right) d^{\prime}\left(\lambda_{1}\right)\right] \cdot\left\langle 0\left|\prod_{j=2}^{N} C\left(\lambda_{j}^{C}\right) \prod_{l=2}^{N} B\left(\lambda_{l}^{B}\right)\right| 0\right\rangle^{\mathrm{mod}} .
$$

Thus we can write down the dependence of the scalar product in the limit (9.1) on the vacuum eigenvalues of argument $\lambda_{1}$ in the explicit form

$$
\begin{aligned}
\left\langle 0\left|\prod_{j=1}^{N} C\left(\lambda_{j}^{C}\right) \prod_{l=1}^{N} B\left(\lambda_{l}^{B}\right)\right| 0\right\rangle= & E \cdot a^{2}\left(\lambda_{1}\right)+F \cdot d^{2}\left(\lambda_{1}\right)+V \cdot a\left(\lambda_{1}\right) d\left(\lambda_{1}\right) \\
& +U \cdot a\left(\lambda_{1}\right) \cdot d\left(\lambda_{1}\right) \frac{d}{d \lambda_{1}} \ln \left[a\left(\lambda_{1}\right) / d\left(\lambda_{1}\right)\right] .
\end{aligned}
$$

The coefficients $E, F, V, U$ are independent of vacuum eigenvalues of argument $\lambda_{1}$. The last one is equal to

$$
U=i \sin 2 \eta\left\langle 0\left|\prod_{j=2}^{N} C\left(\lambda_{j}^{C}\right) \prod_{l=2}^{N} B\left(\lambda_{l}^{B}\right)\right| 0\right\rangle^{\bmod }
$$

[see (9.13), (9.9), (9.10)].

Just now we can do the first step all at one. We let $\lambda_{j}^{C} \rightarrow \lambda_{j}^{D}=\lambda_{j}$. Here all the $\lambda_{j}$ are different. It is easy to check that the results of the previous analysis will not be 
broken down. So the scalar product of the wave function and its dual is equal to

$$
\begin{aligned}
\left\langle 0\left|\prod_{j=1}^{N} C\left(\lambda_{j}\right) \prod_{j=1}^{N} B\left(\lambda_{j}\right)\right| 0\right\rangle= & \tilde{E} \cdot a^{2}\left(\lambda_{1}\right)+\tilde{F} \cdot d^{2}\left(\lambda_{1}\right)+\tilde{V} \cdot a\left(\lambda_{1}\right) d\left(\lambda_{1}\right) \\
& +\tilde{U} a\left(\lambda_{1}\right) d\left(\lambda_{1}\right) \frac{d}{d \lambda_{1}} \ln a\left(\lambda_{1}\right) / d\left(\lambda_{1}\right) .
\end{aligned}
$$

The coefficients $\tilde{E}, \tilde{F}, \tilde{V}$ and $\tilde{U}$ are independent on vacuum eigenvalues of argument $\lambda_{1}$ and

$$
\tilde{U}=i \sin 2 \eta\left\langle 0\left|\prod_{j=2}^{N} C\left(\lambda_{j}\right) \prod_{j=2}^{N} B\left(\lambda_{j}\right)\right| 0\right\rangle^{\mathrm{mod}} .
$$

Here "mod" means (9.10). This is the end of the first step. In the next section we shall do the second step. Namely we shall make $\lambda_{j}$ the solution of the system of TE.

\section{Proof of the Norm Formula for the Eigenstates}

Let us rewrite (9.16) in a form close to (5.1)

$$
\begin{aligned}
\frac{\left\langle 0\left|\prod_{j=1}^{N} C\left(\lambda_{j}\right) \prod_{j=1}^{N} B\left(\lambda_{j}\right)\right| 0\right\rangle}{(\sin 2 \eta)^{N}\left\{\prod_{l=1}^{N} a\left(\lambda_{l}\right) d\left(\lambda_{l}\right)\right\}\left\{\prod_{j \neq K=1}^{N} f\left(\lambda_{j}, \lambda_{K}\right)\right\}} & =E_{1} \frac{a\left(\lambda_{1}\right)}{d\left(\lambda_{1}\right)}+F_{1} \frac{d\left(\lambda_{1}\right)}{a\left(\lambda_{1}\right)} \\
& +V_{1}+U_{1} i \frac{d}{d \lambda_{1}} \ln \frac{a\left(\lambda_{1}\right)}{d\left(\lambda_{1}\right)},
\end{aligned}
$$

where

$$
U_{1}=\frac{\left\langle 0\left|\prod_{j=2}^{N} C\left(\lambda_{j}\right) \prod_{j=2}^{N} B\left(\lambda_{j}\right)\right| 0\right\rangle^{\bmod }}{(\sin 2 \eta)^{N-1}\left\{\prod_{l=2}^{N} \hat{a}\left(\lambda_{l}\right) \hat{d}\left(\lambda_{l}\right)\right\}\left\{\prod_{j \neq K=2}^{N} f\left(\lambda_{j}, \lambda_{K}\right)\right\}} .
$$

Here we use (9.10), (9.9). Now let $\lambda_{j}$ become a solution of the system of TE (2.18). By means of

$$
a\left(\lambda_{1}\right) / d\left(\lambda_{1}\right)=\prod_{j=2}^{N} f\left(\lambda_{j}, \lambda_{1}\right) / f\left(\lambda_{1}, \lambda_{j}\right)
$$

the dependence of the first two terms on the right hand side of $(10.1)$ on $a\left(\lambda_{1}\right)$ and $d\left(\lambda_{1}\right)$ may be eliminated. The dependence on $X_{1}=i d \ln \left[a\left(\lambda_{1}\right) / d\left(\lambda_{1}\right)\right] / d \lambda_{1}[\operatorname{see}(5.3)]$ can not be eliminated by means of TE, see Appendix B. So the value of $\left\|\lambda_{1}, \ldots, \lambda_{N}\right\|_{N}$ in (5.1) depends on vacuum eigenvalues of argument $\lambda_{1}$ only by means of $X_{1}$

where

$$
\left\|\lambda_{1}, \ldots, \lambda_{N}\right\|_{N}=V_{1}+U_{1} X_{1}
$$

$$
U_{1}=\left\|\lambda_{2}, \ldots, \lambda_{N}\right\|_{N-1}^{\bmod } .
$$

Here we use (10.1), (10.2), and (9.10). 
Now we are in a position to check all the five properties of the theorem in Sect. 5. The symmetry property 1 ) is obvious from (2.10). The property 2) is proved by (10.3). The third one is proved by (10.4). The fourth one is proved in Appendix B [see (B.3)]. To prove the fifth one we must consider the limit $\lambda^{B} \rightarrow \lambda^{C}$ in (6.2) and use the definition (5.1). Thus our argument is now complete. We have proved formulas (5.2) and (4.1). The proof of the formula (3.1) is similar. From a formal point of view one does not even need a separate proof as all formulas for the $R$ matrix (2.6) can be obtained directly from formulas for the $R$ matrix (2.5) in the limit (2.7).

\section{Appendix A. Construction of the Quantum Space}

We shall show that a monodromy matrix, which satisfies relation (2.3) with the $R$ matrix (2.8) and possesses arbitrary $a(\lambda)$ and $d(\lambda)$, can be constructed. A nontrivial problem is to construct $T(\lambda)$ which possesses arbitrary ratio $a(\lambda) / d(\lambda)$. Indeed, under multiplication of $T(\lambda)$ by an arbitrary $c$ number function $v(\lambda)$ the vacuum eigenvalues are changed as follows: $a(\lambda) \rightarrow v(\lambda) a(\lambda), d(\lambda) \rightarrow v(\lambda) d(\lambda)$.

In the beginning we shall show that the models known from the literature give us a rich set of $a(\lambda) / d(\lambda)$. First of all let us give an example of two models with the same $R$ matrix but with different vacuum eigenvalues. The monodromy matrix of the lattice sine Gordon model (LSG) is equal to

$$
T(\lambda)=L_{M}(\lambda) \ldots L_{1}(\lambda)
$$

as in (4.5). The statistical weight $L(\lambda)$ was constructed in $[17,18]$. It depends on the spectral parameter $\lambda$, the coupling constant $\eta$ and on the step of the lattice $r$ : $L(\lambda) \equiv L(\lambda, \eta, r)$. The vacuum eigenvalues are equal to

$$
a(\lambda)=[1+2 r \cosh (2 \lambda+2 i \eta)]^{M}, \quad d(\lambda)=[1+2 r \cosh (2 \lambda-2 i \eta)]^{M} .
$$

Thus the monodromy matrices of the 6 vertex model (4.5), (4.6) and of the LSG (A.1) possess the same $R$ matrix (2.8), but different vacuum eigenvalues (4.10) and (A.2).

Let us show that by means of the LSG model we can construct the $T(\lambda)$, for which $a(\lambda) / d(\lambda)$ depends on an infinite amount of parameters. Indeed $T(\lambda)$ for the inhomogeneous lattice is equal to $T(\lambda)=L_{M}\left(\lambda-v_{M}, \eta, r_{M}\right) \ldots L_{1}\left(\lambda-v_{1}, \eta, r_{1}\right)$. Here the spectral parameter and the step of the lattice are site dependent. For this monodromy matrix we have

$$
\frac{a(\lambda)}{d(\lambda)}=\prod_{K=1}^{M}\left[\frac{1+2 r_{K} \cosh \left(2 \lambda+2 i \eta-v_{K}\right)}{1+2 r_{K} \cosh \left(2 \lambda-2 i \eta-v_{K}\right)}\right] .
$$

The $r_{K}$ and $v_{K}, K=1, \ldots, M$ are free parameters. We shall achieve our aim if $M \rightarrow \infty$.

Now let us give the full solution of the problem by means of an algebraic scheme independent of the models. We shall construct the quantum operators $A(\mu), B(\mu), C(\mu)$, and $D(\mu)$ at fixed $a(\lambda)$ and $d(\lambda)$. First we shall construct the space in which these operators act. The basis vectors are

$$
\psi_{N}\left(\left\{\lambda_{j}\right\}\right)=B\left(\lambda_{N}\right) \ldots B\left(\lambda_{1}\right)|0\rangle \text {. }
$$


Here $N$ varies from 0 to $\infty$ and all the $\lambda_{j}$ are free. The whole space consists of the linear combinations of the basis vectors. We shall define all four operators by means of their action in this space. The action of these operators on the basis vectors can be calculated by means of (2.9)-(2.16). Operator $B(\mu)$ acts as a raising operator. The action of the operator $A(\mu)$ is well known [6]

where

$$
\begin{aligned}
A(\mu) B\left(\lambda_{1}\right) \ldots B\left(\lambda_{N}\right)|0\rangle= & \Lambda \cdot B\left(\lambda_{1}\right) \ldots B\left(\lambda_{N}\right)|0\rangle \\
& +\sum_{n=1}^{N} \Lambda_{n} \cdot B(\mu) \prod_{\substack{j=1 \\
j \neq n}}^{N} B\left(\lambda_{j}\right)|0\rangle,
\end{aligned}
$$

$$
\Lambda=a(\mu) \prod_{j=1}^{N} f\left(\mu, \lambda_{j}\right), \quad \Lambda_{n}=a\left(\lambda_{n}\right) g\left(\lambda_{n}, \mu\right) \prod_{\substack{j=1 \\ j \neq n}}^{N} f\left(\lambda_{n}, \lambda_{j}\right),
$$

as well as that of the operator $D(\mu)$

$$
\begin{aligned}
& D(\mu) B\left(\lambda_{1}\right) \ldots B\left(\lambda_{N}\right)|0\rangle= \tilde{\Lambda} \cdot B\left(\lambda_{1}\right) \ldots B\left(\lambda_{N}\right)|0\rangle \\
&+\sum_{n=1}^{N} \tilde{\Lambda}_{n} \cdot B(\mu) \prod_{\substack{j=1 \\
j \neq n}}^{N} B\left(\lambda_{j}\right)|0\rangle, \\
& \tilde{\Lambda}=d(\mu) \prod_{j=1}^{N} f\left(\lambda_{j} \mu\right), \quad \tilde{\Lambda}_{n}=d\left(\lambda_{n}\right) g\left(\mu, \lambda_{n}\right) \prod_{j \neq n} f\left(\lambda_{j}, \lambda_{n}\right) .
\end{aligned}
$$

Operator $C(\mu)$ acts as a lowering operator

$$
C(\mu) \prod_{j=1}^{N} B\left(\lambda_{j}\right)|0\rangle=\sum_{n=1}^{N} M_{n} \prod_{\substack{j \neq n \\ 1}}^{N} B\left(\lambda_{j}\right)|0\rangle+\sum_{K>n} M_{K n} B(\mu) \prod_{\substack{j \neq K, n \\ 1}}^{N} B\left(\lambda_{j}\right)|0\rangle,
$$

where

$$
\begin{aligned}
M_{n}= & g\left(\mu, \lambda_{n}\right) \cdot a(\mu) \cdot d\left(\lambda_{n}\right) \prod_{j \neq n} f\left(\lambda_{j}, \lambda_{n}\right) f\left(\mu, \lambda_{j}\right)+g\left(\lambda_{n}, \mu\right) a\left(\lambda_{n}\right) d(\mu) \\
& \cdot \prod_{j \neq n} f\left(\lambda_{j}, \mu\right) f\left(\lambda_{n}, \lambda_{j}\right), \\
M_{K n}= & d\left(\lambda_{K}\right) \cdot a\left(\lambda_{n}\right) \cdot g\left(\mu, \lambda_{K}\right) \cdot g\left(\lambda_{n}, \mu\right) \cdot f\left(\lambda_{n}, \lambda_{K}\right)\left\{\prod_{j \neq K, n}^{N} f\left(\lambda_{j}, \lambda_{K}\right) f\left(\lambda_{n}, \lambda_{j}\right)\right\} \\
& +d\left(\lambda_{n}\right) a\left(\lambda_{K}\right) g\left(\mu, \lambda_{n}\right) g\left(\lambda_{K}, \mu\right) f\left(\lambda_{K}, \lambda_{n}\right)\left\{\prod_{j \neq K, n}^{N} f\left(\lambda_{j}, \lambda_{n}\right) f\left(\lambda_{K}, \lambda_{j}\right)\right\} .
\end{aligned}
$$

In this way the action of our operators on any vector in the quantum space is defined. By means of direct calculations one can show that all equalities containing in the relation (2.3) are valid.

The dual vacuum $\langle 0|$ can be defined as a linear functional in the space constructed above. The main properties of this functional are as follows:

$$
\langle 0 \mid 0\rangle=1, \quad\left\langle 0\left|\prod_{j=1}^{N} B\left(\lambda_{j}\right)\right| 0\right\rangle=0, \quad \text { for } \quad N \geqq 1 .
$$

Then the second line of (2.16) can be simply proved. Commutation relations (2.10), (2.13); (2.14), and (2.16) permit one to prove (2.21). 


\section{Appendix B. The Norms at $X_{p}=0$}

Notice that in Appendix A we showed that operators $A(\mu), B(\mu), C(\mu), D(\mu)$ can be realized in the case of arbitrary $a(\lambda)$ and $d(\lambda)$. Let $\lambda_{j}$ be a solution of TE (2.18). We shall show that the variables

$$
X_{K}=i \frac{d}{d \lambda_{K}} \ln \frac{a\left(\lambda_{K}\right)}{d\left(\lambda_{K}\right)}
$$

are $\lambda_{j}$ independent. They can change freely at fixed $\lambda_{j}$. Indeed, let us choose the new function $\tilde{a}(\lambda) / \tilde{d}(\lambda)$, which coincides with old one only in the points $\lambda_{j}$ : $a\left(\lambda_{n}\right) / d\left(\lambda_{n}\right)=\tilde{a}\left(\lambda_{n}\right) / \tilde{d}\left(\lambda_{n}\right)$. The set of $\lambda_{j}$ satisfy also a new system of TE

$$
\left[\tilde{a}\left(\lambda_{n}\right) / \tilde{d}\left(\lambda_{n}\right)\right] \prod_{\substack{j=1 \\ j \neq n}}^{N}\left[f\left(\lambda_{n}, \lambda_{j}\right) / f\left(\lambda_{j}, \lambda_{n}\right)\right]=1,
$$

but the variables $X_{K}$ are changed.

Let us use this transformation to prove (5.14)

$$
\left\|\lambda_{1}, \ldots, \lambda_{N}\right\|_{N}=0 \quad \text { at } \quad X_{p}=0, \quad p=1, \ldots, N .
$$

We construct the new function as follows:

$$
\tilde{a}(\lambda) / \tilde{d}(\lambda)=a\left(\lambda_{j}\right) / d\left(\lambda_{j}\right), \quad \text { if } \quad \lambda_{j}-S<\lambda<\lambda+S .
$$

It is constant in some vicinity of each $\lambda_{j}$. Since all the $\lambda_{j}$ are different, let us choose

$$
4 S=\min _{j \neq K}\left|\lambda_{j}-\lambda_{K}\right| .
$$

In other regions $\tilde{a}(\lambda) / \tilde{d}(\lambda)$ must be smooth (see Fig. 1). Let us calculate the $\left\|\lambda_{1}, \ldots, \lambda_{N}\right\|_{N}$ for the vacuum eigenvalues. As all $X_{K}=0$, now, we have

$$
\left\|\lambda_{1} \ldots \lambda_{N}\right\|^{\text {new }}=\left.\left\|\lambda_{1} \ldots \lambda_{N}\right\|^{\text {old }}\right|_{X_{p}=0} .
$$

So to prove (B.3) it is sufficient to show that the scalar product of the eigenfunction and its dual

$$
\left\langle 0\left|C\left(\lambda_{1}\right) \ldots C\left(\lambda_{N}\right) B\left(\lambda_{1}\right) \ldots B\left(\lambda_{N}\right)\right| 0\right\rangle=0
$$

for the new values $\tilde{a}(\lambda)$ and $\tilde{d}(\lambda)$. Notice that now not only the $\lambda_{j}$ are solutions of (B.2) but

$$
\lambda_{j}(y)=\lambda_{j}+y, \quad-S<y<S
$$

Fig. 1

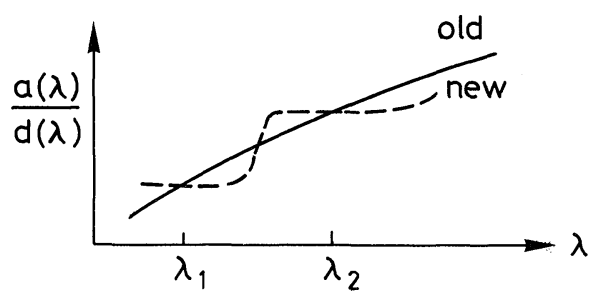


are also solutions of (B.2), due to the fact that $f\left(\lambda_{n}, \lambda_{j}\right)$ depends only on the difference $\lambda_{n}-\lambda_{j}$ and $\tilde{a}(\lambda) / \tilde{d}(\lambda)$ are constants in this region. We shall see that the scalar product

$$
\left\langle 0\left|\prod_{j=1}^{N} C\left(\lambda_{j}\right) \prod_{j=1}^{N} B\left(\lambda_{j}+y\right)\right| 0\right\rangle \equiv G(y)
$$

is equal to zero at any $0<y<S,-S<y<0$. Indeed, the object

$$
\left\langle 0\left|\prod_{j=1}^{N} C\left(\lambda_{j}\right)[A(\mu)+D(\mu)] \prod_{j=1}^{N} B\left(\lambda_{j}+y\right)\right| 0\right\rangle
$$

can be calculated in two ways. Either $[A(\mu)+D(\mu)]$ goes to the left or to the right. As both states are eigenstates we have two expressions for (B.10)

where

$$
\theta\left(\mu,\left\{\lambda_{j}\right\}\right) G(y), \text { or } \theta\left(\mu,\left\{\lambda_{j}+y\right\}\right) \cdot G(y),
$$

$$
\theta\left(\mu,\left\{\lambda_{j}\right\}\right)=a(\mu) \prod_{j=1}^{N} f\left(\mu, \lambda_{j}\right)+d(\mu) \prod_{j=1}^{N} f\left(\lambda_{j}, \mu\right),
$$

see (2.19). As $\theta\left(\mu,\left\{\lambda_{j}\right\}\right) \neq \theta\left(\mu,\left\{\lambda_{j}+y\right\}\right)$ at $y \neq 0$ we have $G(y)=0$ at $y \neq 0$. As the scalar product (B.9) depends on $y$ in a continuous way we have $G(0)=0$ and therefore (B.7), (B.3), and (5.14) is valid.

\section{Appendix C. Eigenvalue of $S_{3}$ on the Bethe States}

We discuss the inhomogeneous 6 vertex model. The operator of the third component of the total spin

$$
S^{3}=\sum_{K=1}^{M} \sigma_{K}^{3}
$$

is important. The pseudovacuum (4.8) and the state $\Omega(7.6)$ are its eigenstates

$$
S^{3}|0\rangle=M|0\rangle, \quad S^{3} \Omega=-M \Omega .
$$

Any state (A.1) with free $\lambda_{j}$ is an eigenstate of $S^{3}$

$$
S^{3} \prod_{j=1}^{M} B\left(\lambda_{j}\right)|0\rangle=(M-2 N)|0\rangle .
$$

See for example [22]. For completeness we shall give the proof. We use the identity

and locality

$$
\left[L_{K}(\lambda), \sigma^{3}+\sigma_{K}^{3}\right]=0
$$

$$
\left[L_{K}(\lambda), \sigma_{l}^{3}\right]=0 \quad \text { at } \quad K \neq l .
$$

Let us calculate the commutator

$$
\begin{aligned}
{\left[T(\lambda), S^{3}\right] } & =\sum_{K=1}^{M} L_{M} L_{M-1} \ldots L_{K+1}\left[L_{K}, \sigma_{K}^{3}\right] L_{K-1} \ldots L_{1} \\
& =-\sum_{K=1}^{M} L_{M} \ldots\left[L_{K}, \sigma^{3}\right] \ldots L_{1}=-\left[T(\lambda), \sigma^{3}\right] .
\end{aligned}
$$


Here $\sigma^{3}$ is not a spin operator but a $2 \times 2$ matrix. From (C.6) we have

$$
\left[B(\lambda), S^{3}\right]=2 B(\lambda) .
$$

Combination of this formula and (C.2) gives us (C.3). Let the number of $B(\lambda)$ be equal to the number of the sites in the lattice $N=M$. Then (C.3) gives

$$
S^{3} \prod_{j=1}^{M} B\left(\lambda_{j}\right)|0\rangle=-M|0\rangle
$$

Comparison with (C.2) leads to

$$
\prod_{j=1}^{M} B\left(\lambda_{j}\right)|0\rangle=Z_{N} \cdot \Omega
$$

Here $Z_{N}$ is a $c$ number.

\section{Appendix D. Construction of the General Version of the 6 Vertex Model}

The inhomogeneous 6 vertex model has the following weight matrix:

$$
\begin{aligned}
L_{\alpha K}\left(\lambda_{\alpha}-v_{K}\right)=\cos \eta \cdot \sinh \left(\lambda_{\alpha}-v_{K}\right) & -i \sin \eta \cdot \cosh \left(\lambda_{\alpha}-v_{K}\right) \sigma_{\alpha}^{3} \sigma_{K}^{3} \\
& -i \sin 2 \eta\left(\sigma_{\alpha}^{-} \sigma_{K}^{+}+\sigma_{\alpha}^{+} \sigma_{K}^{-}\right) .
\end{aligned}
$$

This statistical weight corresponds to the lattice vertex with the same index (see Fig. 2). The matrix indices of the $\alpha^{\text {th }}$ space correspond to spins on the horizontal edges $\left(a_{\alpha}, b_{\alpha}\right)$, while the matrix indices of the $K^{\text {th }}$ space correspond to spins on the

Fig. 2

$$
a_{\alpha}-\left.\right|_{b_{k}} ^{a_{k}} b_{\alpha} \quad\left(L_{\alpha k}\right) a_{a_{\alpha} b_{\alpha}}^{a_{k} b_{k}}
$$

vertical edges $a_{K}, b_{K}$. Before constructing the model itself we shall mention some properties of the $L$ matrix. Its commutation relations are given by (2.3)

$$
R_{\alpha \beta}\left(\lambda_{\alpha}, \lambda_{\beta}\right) L_{\alpha K}\left(\lambda_{\alpha}-v_{K}\right) L_{\beta K}\left(\lambda_{\beta}-v_{K}\right)=L_{\beta K}\left(\lambda_{\beta}-v_{K}\right) L_{\alpha K}\left(\lambda_{\alpha}-v_{K}\right) R_{\alpha \beta}\left(\lambda_{\alpha}, \lambda_{\beta}\right),
$$

with $R$ matrix (2.8). One can change the position of the vertical and horizontal spaces

$$
R_{K l}\left(v_{l}, v_{K}\right) L_{\alpha K}\left(\lambda_{\alpha}-v_{K}\right) L_{\alpha l}\left(\lambda_{\alpha}-v_{l}\right)=L_{\alpha l}\left(\lambda_{\alpha}-v_{l}\right) L_{\alpha K}\left(\lambda_{\alpha}-v_{K}\right) R_{K l}\left(v_{l} v_{K}\right) .
$$

If $v_{K}-\lambda_{\alpha}=i \eta$, the $L$ matrix is especially simple

$$
L_{\alpha K}\left(v_{K}-\lambda_{\alpha}=i \eta\right)=-i \sin 2 \eta \Pi_{\alpha K} .
$$

Here $\Pi$ is the permutation matrix

$$
\Pi_{\alpha K}\left(\vec{v}_{K} \vec{w}_{\alpha}\right)=\vec{w}_{K} \cdot \vec{v}_{\alpha} .
$$


Here $\vec{v}$ and $\vec{w}$ are two-component $c$-number vectors. The index shows the space in which this vector is situated. Then the $L$ matrix (D.1) has two simple eigenvectors

$$
\begin{aligned}
& L_{\alpha K}\left(\lambda_{\alpha}-v_{K}\right)\left(\uparrow_{\alpha} \uparrow_{K}\right)=\sinh \left(\lambda_{\alpha}-v_{K}-i \eta\right)\left(\uparrow_{\alpha} \uparrow_{K}\right) \\
& L_{\alpha K}\left(\lambda_{\alpha}-v_{K}\right)\left(\downarrow_{\alpha} \downarrow_{K}\right)=\sinh \left(\lambda_{\alpha}-v_{K}-i \eta\right)\left(\downarrow_{\alpha} \downarrow_{K}\right) .
\end{aligned}
$$

Here we use the notation (4.9). The last property of $L$ is

$$
\left[L_{\alpha K}\left(\lambda_{\alpha}-v_{K}\right), \sigma_{K}^{1} \sigma_{\alpha}^{1}\right]=0 .
$$

We shall construct the inhomogeneous 6 vertex model following [23]. Let us consider an inhomogeneous square lattice with $N$ lines and $N$ columns (see Fig. 3).

Fig. 3

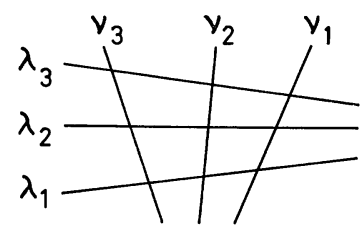

Each $\lambda_{\alpha}$ corresponds to a horizontal line, and each $v_{K}$ corresponds to a vertical line. The statistical weight (D.1) is associated to the intersection of the $\alpha^{\text {th }}$ and $K^{\text {th }}$ lines. The summation is carried out over the spins on the interior edges. This summation is reduced to the matrix multiplication of the $L$ operators in the corresponding spaces. The spins on the boundary of the lattice are fixed. The boundary consists of four lines. We denote spins on each of these lines by $\vec{u}\left(v_{j}\right)$, $\vec{r}\left(\lambda_{\alpha}\right), \vec{d}\left(v_{j}\right), \vec{l}\left(\lambda_{\alpha}\right)$ correspondingly (see Fig. 4).

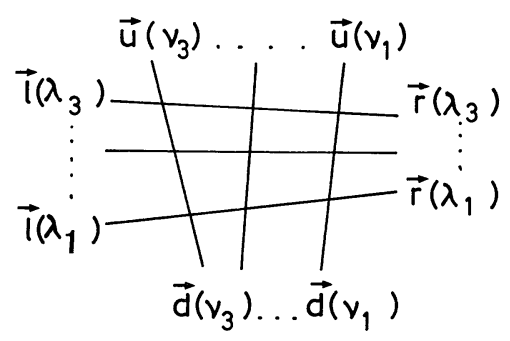

Fig. 4

The partition function is equal to:

$$
\begin{aligned}
Z_{N}\left(\left\{\lambda_{\alpha}\right\},\left\{v_{K}\right\}\right)= & \left\{\prod_{\alpha=1}^{N} \vec{l}\left(\lambda_{\alpha}\right)\right\}\left\{\prod_{j=1}^{N} \vec{u}\left(v_{j}\right)\right\} \\
& \cdot\left\{\prod_{\alpha=1}^{\overleftarrow{N}} \prod_{K=1}^{\overleftarrow{N}} L_{\alpha K}\left(\lambda_{\alpha}-v_{K}\right)\right\}\left\{\prod_{\alpha=1}^{N} \vec{r}\left(\lambda_{\alpha}\right)\right\}\left\{\prod_{j=1}^{N} \vec{d}\left(v_{j}\right)\right\} .
\end{aligned}
$$

In this product $L_{\alpha K}$ stands to the left of $L_{\beta l}$ if $N \alpha+K>N \beta+l$. If $\alpha \neq \beta$ and $K \neq l$ these $L$ commute. If $\alpha=\beta$ or $K=l$ they are multiplied as matrices in this space. We 
shall use the transfer matrix method to rearrange the partition function. Let us collect the $L$ matrixes along the horizontal lines

$$
T_{\alpha}\left(\lambda_{\alpha}\right)=\prod_{K=1}^{\overleftarrow{N}} L_{\alpha K}\left(\lambda_{\alpha}-v_{K}\right)=\left(\begin{array}{ll}
A\left(\lambda_{\alpha}\right) & B\left(\lambda_{\alpha}\right) \\
C\left(\lambda_{\alpha}\right) & D\left(\lambda_{\alpha}\right)
\end{array}\right)
$$

Here $T_{\alpha}$ is represented as an explicit $2 \times 2$ matrix in $\alpha^{\text {th }}$ space. This $T$ coincides exactly with the monodromy matrix (7.2), (7.1). In terms of $T_{\alpha}$ the partition function is equal to

$$
Z_{N}=\left\{\prod_{j=1}^{N} \vec{u}\left(v_{j}\right)\right\} \prod_{\alpha=1}^{\overleftarrow{N}}\left(\vec{l}\left(\lambda_{\alpha}\right) T_{\alpha}\left(\lambda_{\alpha}\right) \vec{r}\left(\lambda_{\alpha}\right)\right)\left\{\prod_{j=1}^{N} \vec{d}\left(v_{j}\right)\right\} .
$$

Notice that $\vec{l}\left(\lambda_{\alpha}\right) T_{\alpha}\left(\lambda_{\alpha}\right) \vec{r}\left(\lambda_{\alpha}\right)$ is a scalar in the $\alpha$ space. To have another representation for $Z_{N}$ let us collect the $L$ matrixes along the vertical lines

$$
\tau_{j}\left(v_{j}\right)=\prod_{\alpha=1}^{\overleftarrow{N}} L_{\alpha j}\left(\lambda_{\alpha}-v_{j}\right)=\left(\begin{array}{ll}
A\left(v_{j}\right) & B\left(v_{j}\right) \\
C\left(v_{j}\right) & D\left(v_{j}\right)
\end{array}\right) .
$$

Here $\tau_{j}$ is represented as an explicit $2 \times 2$ matrix in $j$ space. The commutation relations of $\tau_{j}$ are

$$
R_{K l}\left(v_{l}, v_{K}\right) \tau_{K}\left(v_{K}\right) \tau_{l}\left(v_{l}\right)=\tau_{l}\left(v_{l}\right) \tau_{\boldsymbol{K}}\left(v_{K}\right) R_{K l}\left(v_{l}, v_{K}\right)
$$

with the $R$ matrix (2.8), due to (D.3). In terms of $\tau\left(v_{j}\right)$ the partition function is equal to:

$$
Z_{N}=\left\{\prod_{\alpha=1}^{N} \vec{l}\left(\lambda_{\alpha}\right)\right\}\left\{\prod_{j=1}^{\overleftarrow{N}} \vec{u}\left(v_{j}\right) \tau_{j}\left(v_{j}\right) \vec{d}\left(v_{j}\right)\right\}\left\{\prod_{\alpha=1}^{N} \vec{r}\left(\lambda_{\alpha}\right)\right\}
$$

The following boundary conditions are of special interest for us:

$$
\begin{array}{ll}
\vec{l}\left(\lambda_{\alpha}\right)=\uparrow_{\alpha}, \quad \vec{u}\left(v_{K}\right)=\downarrow_{K} \\
\vec{r}\left(\lambda_{\alpha}\right)=\downarrow_{\alpha}, \quad \vec{d}\left(v_{K}\right)=\uparrow_{K} .
\end{array}
$$

In this case

$$
Z_{N}=\left(\prod_{j=1}^{N} \downarrow_{j}\right)\left(\prod_{\alpha=1}^{N} \uparrow_{\alpha}\right)\left\{\prod_{\alpha=1}^{\overleftarrow{N}} \prod_{K=1}^{\overleftarrow{N}} L_{\alpha K}\left(\lambda_{\alpha}-v_{K}\right)\right\}\left(\prod_{j=1}^{N} \uparrow_{j}\right)\left(\prod_{\alpha=1}^{N} \downarrow_{\alpha}\right)
$$

Due to (D.10) we have

$$
Z_{N}\left(\left\{\lambda_{\alpha}\right\},\left\{v_{j}\right\}\right)=\Omega B\left(\lambda_{N}\right) \ldots B\left(\lambda_{1}\right)|0\rangle .
$$

Here we use $\uparrow_{\alpha} T_{\alpha}(\lambda) \downarrow_{\alpha}=B(\lambda)$ and notations (4.8), (7.6). Notice that (D.16) and (7.7) coincide, as the number of the sites in the lattice coincides with the number of $B(\lambda)$. One can show that

$$
Z_{N}\left(\left\{\lambda_{\alpha}\right\},\left\{v_{j}\right\}\right)=\langle 0| C\left(\lambda_{1}\right) \ldots C\left(\lambda_{N}\right) \Omega
$$


Indeed, let us introduce the operator

$$
U=\prod_{\alpha=1}^{N}\left(\sigma_{\alpha}^{1}\right) \prod_{K=1}^{N} \sigma_{K}^{1}
$$

This operator has two properties:

$$
\left[U, \prod_{\alpha=1}^{\overleftarrow{N}} \prod_{K=1}^{\overleftarrow{N}} L_{\alpha K}\left(\lambda_{\alpha}-v_{K}\right)\right]=0, \quad U^{2}=1
$$

Here we use (D.7). So

$$
\begin{aligned}
Z_{N} & =\left(\prod \downarrow_{j}\right)\left(\prod \uparrow_{\alpha}\right) U\left\{\prod_{\alpha}^{\leftarrow} \prod_{K} L_{\alpha K}\right\} U\left(\prod \uparrow_{j}\right)\left(\prod \downarrow_{\alpha}\right) \\
& =\left(\prod \uparrow_{j}\right)\left(\prod \downarrow_{\alpha}\right)\left\{\prod_{\alpha}^{\leftarrow} \prod_{K}^{\leftarrow} L_{\alpha K}\right\}\left(\prod \downarrow_{j}\right)\left(\prod \uparrow_{\alpha}\right),
\end{aligned}
$$

which leads to (D.17). To manifest the $v_{j}$ dependence of $Z_{N}$, let us use representation (D.13). By means of $\downarrow_{j} \tau_{j}(v) \uparrow_{j}=C(v)$ we obtain

$$
Z_{N}\left(\left\{\lambda_{\alpha}\right\},\left\{v_{j}\right\}\right)=\langle 0| C\left(v_{N}\right) \ldots C\left(v_{1}\right) \Omega .
$$

This means that $Z_{N}$ is symmetric in all $v_{j}$ due to (2.10).

Let us show at last that if $v_{1}-\lambda_{1}=i \eta$, then $Z_{N}$ is reduced to $Z_{N-1}$ with $\lambda_{1}$ and $v_{1}$ removed. We have by means of (D.4)

$$
L_{11}\left(v_{1}-\lambda_{1}=i \eta\right)=-i \cdot \sin 2 \eta \cdot \Pi_{11} .
$$

Notice that this $L$ is the one farthest to the right in the product (D.15). Let us calculate

$$
L_{11}\left(\prod_{j=1}^{N} \uparrow_{j}\right)\left(\prod_{\alpha=1}^{N} \downarrow_{\alpha}\right)=-i \sin 2 \eta\left(\downarrow_{1} \prod_{j=2}^{N} \uparrow_{j}\right)\left(\uparrow_{1} \prod_{\alpha=2}^{N} \downarrow_{\alpha}\right) .
$$

The vector on the right hand side is the eigenstate of all the $L_{1 j}\left(\lambda_{1}-v_{j}\right), j=2, \ldots, N$, see (D.6). It is also an eigenstate of all $L_{\alpha 1}\left(\lambda_{\alpha}-v_{1}\right), \alpha=2, \ldots, N$ for the same reason. After application of all $L_{1 j}$ and $L_{\alpha 1}$ to the right hand side of (D.23) we have

$$
\begin{aligned}
\left.Z_{N}\left(\left\{\lambda_{\alpha}\right\},\left\{v_{j}\right\}\right)\right|_{v_{1}-\lambda_{1}=i \eta}= & -i \sin 2 \eta\left\{\prod_{K=2}^{N} \sinh \left(\lambda_{1}-v_{K}-i \eta\right)\right\} \\
& \cdot\left\{\prod_{\alpha=2}^{N} \sinh \left(\lambda_{\alpha}-v_{1}-i \eta\right)\right\} \cdot Z_{N-1}\left(\left\{\lambda_{\alpha \neq 1}\right\},\left\{v_{j \neq 1}\right\}\right) .
\end{aligned}
$$

\section{Appendix E. Properties of the Scalar Products}

In Sect. 6 the properties of the coefficients in the formula for the scalar product (6.7) are given. Here we shall give some corollaries. Let $\left\{\lambda^{A C}\right\}$ and $\left\{\lambda^{D B}\right\}$ be 
nonempty sets as in (9.2), (9.3). Let us study the limit

$$
\lambda_{1}^{A C}=\lambda_{1}^{D B}=\lambda_{1}
$$

Then from (6.19) and (6.16) we have

$$
\begin{aligned}
\pi_{N}\left(\begin{array}{ll}
\left\{\lambda_{j}^{C}\right\} & \left\{\lambda_{j}^{B}\right\} \\
\left\{\lambda_{j}^{A}\right\} & \left\{\lambda_{j}^{D}\right\}
\end{array}\right)=(-i \sin 2 \eta)\left(\prod_{j=2}^{N} \sinh \left(\lambda_{j}^{D}-\lambda_{1}-2 i \eta\right) \sinh \left(\lambda_{1}-\lambda_{j}^{A}-2 i \eta\right)\right) \\
\cdot \pi_{N-1}\left(\begin{array}{ll}
\left\{\lambda_{j \neq 1}^{C}\right\} & \left\{\lambda_{j \neq 1}^{B}\right\} \\
\left\{\lambda_{j \neq 1}^{A}\right\} & \left\{\lambda_{j \neq 1}^{D}\right\}
\end{array}\right) .
\end{aligned}
$$

Similarly if $\left\{\lambda^{A B}\right\} \neq \emptyset$ and $\left\{\lambda^{D C}\right\} \neq \emptyset$ we shall use the following enumeration:

$$
\lambda_{1}^{C}=\lambda_{1}^{D}=\lambda_{1}^{D C}, \quad \lambda_{1}^{A}=\lambda_{1}^{B}=\lambda_{1}^{A B} .
$$

The interesting case is

$$
\lambda_{1}^{D C}=\lambda_{1}^{A B}=\lambda_{1}
$$

The formulas (6.19) and (6.16) lead to

$$
\begin{gathered}
\pi_{N}\left(\begin{array}{ll}
\left\{\lambda_{j}^{C}\right\} & \left\{\lambda_{j}^{B}\right\} \\
\left\{\lambda_{j}^{A}\right\} & \left\{\lambda_{j}^{D}\right\}
\end{array}\right)=(-i \sin 2 \eta)\left(\prod_{j=2}^{N} \sinh \left(\lambda_{1}-\lambda_{j}^{A}-2 i \eta\right) \sinh \left(\lambda_{j}^{D}-\lambda_{1}-2 i \eta\right)\right) \\
\cdot \pi_{N-1}\left(\begin{array}{ll}
\left\{\lambda_{j \neq 1}^{C}\right\} & \left\{\lambda_{j \neq 1}^{B}\right\} \\
\left\{\lambda_{j \neq 1}^{A}\right\} & \left\{\lambda_{j \neq 1}^{D}\right\}
\end{array}\right) .
\end{gathered}
$$

Acknowledgements. I thank L. D. Faddeev for useful discussions. I want to emphasize the stimulating influence of M. Gaudin's hypothesis about the norms of eigenfunctions in the nonlinear Schrödinger equation. I also appreciate the help of J. Lowenstein, H. de Vega, M. Semenov-Tian-Shansky and D. Scott. I am grateful to the Service de Physique Théorique de CEN de Saclay for hospitality.

\section{References}

1. Bethe, H.: Z. Phys. 71, 205-226 (1931)

2. Yang, C.N., Yang, C.P. : Phys. Rev. 150, 321-327 (1966)

3. Lieb, E.H.: Phys. Rev. Lett. 18, 692-694 (1967)

4. Berezin, F.A., Pokhil, C.P., Finkelberg, V.M.: Vestn. Mosk. Gos. Univ. Ser. 1.1, 21-28 (1964)

5. McGuire, J.B. : J. Math. Phys. 5, 622-636 (1964); Brézin, E., Zinn-Justin, J.: C.R. Acad. Sci. Paris 263, 670-673 (1966);

Gaudin, M.: J. Math. Phys. 12, 1674-1676, 1677-1680 (1971)

6. Faddeev, L.D.: Sov. Sci. Rev. Math. Phys. C1, 107-160 (1981)

7. Faddeev, L.D., Takhtajan, L.A. : Usp. Mat. Nauk 34, 13-63 (1979)

8. Gaudin, M.: Preprint, Centre d'Etudes Nucleaires de Saclay, CEA-N-1559, (1), (1972)

9. Gaudin, M., McCoy, B.M., Wu, T.T.: Phys. Rev. D 23, 417-419 (1981)

10. Baxter, R.J.: J. Stat. Phys. 9, 145-182 (1973)

11. Izergin, A.G., Korepin, V.E.: Lett. Math. Phys. (to be published)

12. Faddeev, L.D., Sklyanin, E.K.: Dokl. Akad. Nauk SSSR 243, 1430-1433 (1978)

13. Sklyanin, E.K.: Dokl. Akad. Nauk SSSR 244, 1337-1341 (1978)

14. Izergin, A.G., Korepin, V.E., Smirnov, F.A.: Teor. Mat. Fiz. 48, 319-323 (1981)

15. Sklyanin, E.K.: Zap. Nauchn. Seminarov LOMI 95, 55-128 (1980)

16. Izergin, A.G., Korepin, V.E.: Dokl. Akad. Nauk SSSR 259, 76-79 (1981) 
17. Izergin, A.G., Korepin, V.E.: Nucl. Phys. B, Field Theory and Statistical Systems B 205 [FS5], 401-413 (1982)

18. Izergin, A.G., Korepin, V.E.: Lett. Math. Phys. 5, 199-205 (1981)

19. Baxter, R.J.: Stud. Appl. Math. L 50, 51-67 (1971)

20. Belavin, A.A.: Phys. Lett. B 87, 117-121 (1980)

21. Kulish, P.P.: Physica D 3, 246-257 (1981)

22. Faddeev, L.D., Takhtajan, L.A.: Zap. Nauchn. Seminarov LOMI 109, 134-178 (1981)

23. Baxter, R.J.: Philos. Trans. Soc. London, Ser. A 289, 315-346 (1978)

Communicated by A. Jaffe

Received January 27, 1982; in revised form May 10, 1982 